\title{
A Novel, Personalized Drug-Screening System for Platinum-Resistant Ovarian Cancer Patients: A Preliminary Clinical Report
}

This article was published in the following Dove Press journal: Cancer Management and Research

\author{
Yunke Huang $\mathbb{D}^{*}$ \\ Jing $\mathrm{Xu}^{*}$ \\ $\mathrm{Ke} \mathrm{Li}$ \\ Jing Wang \\ Yilin Dai \\ Yu Kang
}

The Obstetrics \& Gynecology Hospital of Fudan University, Gynecology Department, Shanghai, People's Republic of China

*These authors contributed equally to this work
Correspondence: Yu Kang

The Obstetrics \& Gynecology Hospital of Fudan University, Gynecology

Department, No. 419, Fangxie Road,

Huangpu District, Shanghai, People's

Republic of China

Tel +86 I36 363282II

Email yukang@fudan.edu.cn
Purpose: With this study, we intended to construct a personalized drug-screening system for platinum-resistant ovarian cancer patients by consulting a patient's medical history, data derived from gene mutation detection, and drug screening results derived from mini-PDX (patient-derived xenograft) models. We also aimed to evaluate the efficacy and safety of our system.

Patients and Methods: We selected 12 patients with platinum-resistant ovarian cancer who were treated at our hospital from January 2018 to December 2019 to design a single-arm clinical trial. The subsequent chemotherapeutic plans were selected according to a personalized drug-screening system that circulating tumor DNA (ctDNA) testing and the establishment of mini-PDX models. We then analyzed the patients for clinical benefits side-effects in response to chemotherapy in order to evaluate the clinical effects and safety of our new personalized drug-selection system.

Results: We successfully established an individualized and sensitive drug-screening system for the 12 patients. Mini-PDX models verified that potentially effective drugs were identified for 11 of the patients. Treatment resulted in complete remission (one patient), partial remission (five patients), and stable disease (three patients). The remaining three patients experienced disease progression. The overall clinical-benefit rate was $75.0 \%$. Following treatment, the levels of CA125 levels decreased significantly in seven of the 12 patients. Severe side effects, arising from chemotherapy, were only observed in one case.

Conclusion: Constructing a personalized drug-screening system for platinum-resistant ovarian cancer patients can be used to guide clinical drug selection and improve the clinical-benefit rate for patients.

Trial Registration Number: ChiCTR1800016766 (Chinese Clinical Trial Registry Center).

Keywords: drug resistance, ovarian cancer, precision medicine, personalized drug-screening system

\section{Introduction}

Ovarian cancer is the deadliest form of gynecological cancer. The standard form of treatment for this cancer is tumor load-reducing surgery combined with subsequent platinum-based chemotherapy. ${ }^{1}$ However, $70 \%$ of patients who reach complete remission (CR) after primary systemic treatment will experience the recurrence of ovarian cancer within 3 years. Furthermore, the proportion of platinum-resistant cancer (recurrence within 6 months of the last treatment cycle $^{2}$ ) is significantly higher, thus resulting in a 5-year overall survival (OS) of only $20 \%$ to $30 \%{ }^{3,4}$ For 
platinum-resistant patients, the median survival time is only 12 months while the median progression-free survival (PFS) is only 4-5 months. ${ }^{5,6}$ The National Comprehensive Cancer Network (NCCN) guidelines recommend over 10 types of second-line, single-drug chemotherapy plans for platinum-resistant disease, including docetaxel, oral etoposide, weekly paclitaxel (with or without pazopanib), liposomal doxorubicin (with or without bevacizumab), weekly bevacizumab, topotecan (with or without bevacizumab), bevacizumab + oral cyclophosphamide; and three targettherapy plans (bevacizumab, olaparib, and rucaparib). All of these chemotherapy plans show similar and unsatisfactory objective response rates (ORRs) $(15 \%-30 \%) .^{7-13}$

One solution to improve treatment for platinum-resistant ovarian cancer would be the development of precision medicine. ${ }^{14-16}$ Since the odds associated with this type of cancer are unsatisfactory, a personalized therapy plan designed to incorporate gene-testing technology might improve the situation. ${ }^{17-21}$ In addition, functional diagnoses could also help to predict the chemotherapy plan that would have the greatest effects on patients. Patientderived xenograft (PDX) models ${ }^{22,23}$ are constructed by directly implanting patient tumor fragments into immunodeficient mice; the mice can then be tested with a variety of anti-cancer regimens. Drug responses can then be evaluated by determining the weights of tumors harvested from the mice after 30-40 days of observation. PDX models have been used on over 10 types of solid tumors and are capable of reflecting the clinical effects of different chemotherapeutic drugs with a $70 \%-100 \%$ concordance rate between patient clinical response and the therapeutic response of the PDX model. However, the procedural establishment of this model normally requires 4-8 months, thus making it impossible for these models to guide clinical treatment. ${ }^{24-27}$ More recently, mini-PDX models have been developed; these models generate drug-sensitivity test results within 7-10 days. This drug-response assay was first reported in 2018 by Zhang, ${ }^{28}$ who established this model by placing tumor fragments from patients into a hollow fiber-culture system and then implanting the fiber capsules subcutaneously into immunodeficient mice. In another study, Ming's team demonstrated that the use of a mini-PDX system to assist in drug-selection procedures provided notable benefit to patients with gallbladder cancer. $^{29}$

In the present study, we aimed to establish an individualized drug-screening system by combining treatmenthistory analysis, mutation detection, and the establishment of mini-PDX models. We hypothesize that this system could provide patients with the most-effective individualized treatment plan in order to prolong patient survival.

\section{Patients and Methods Clinical Protocol}

We enrolled 12 patients with platinum-resistant ovarian cancer into our study from January 2018 to December 2019. Next, we designed a personalized drug-screening system for these patients in a single-arm clinical study that combined their medical history and mutation detection results. The regimens that exhibited high probabilities of inhibiting tumor growth in mini-PDX models were then used for clinical treatment.

The personalized drug-screening system was established via three main processes: the collection of patient information, gene mutation detection, and the establishment of mini-PDX models.

\section{Collection of Patient Information}

We collected a range of basic information from each patient, including a detailed medical history, surgical information, pathological findings, imaging examination results, and the patient's family history of malignant tumors.

\section{Gene Mutation Detection}

We collected $10 \mathrm{~mL}$ of blood from each patient to carry out circulating tumor DNA (ctDNA) tests; we needed this data to establish a specific mini-PDX system. The ctDNA tests were carried out at the central laboratory of Topgen Bio-Pharm Co, Ltd. Next Generation Sequencing(NGS) was carried out with an OncoDrug-Seq ${ }^{\mathrm{TM}}$ (TopgenBiopharm, Shanghai, China) for a panel of 81 tumortargeting genes including $B R C A 1, B R C A 2, K R A S$, and TP53. Sanger sequencing was also performed to classify germline and somatic mutations after they had been detected by NGS.

ctDNA was isolated using the Qiagen QIAamp Circulating Nucleic Acid Kit (Qiagen, Cat\# 55,114), following the manufacturer's protocol. A Qubit dsDNA HS Assay Kit (Thermo Fisher Scientific, Cat\# Q32854) was then used to determine the concentration of ctDNA samples. The quality of each ctDNA sample was then determined with an Agilent High Sensitivity DNA Kit (Cat\# 5067-4626). Library construction was carried out using 
concentrated ctDNA ( $>5 \mathrm{ng}$ without excessive genomic DNA contamination) and the KAPA HyperPlus Kit (KAPA Biosystems, Cat\# KK8504). Targeted enrichment was performed using a Target Probes IGT Kit (iGene Tech, cat\# T232 V2) and NGS sequencing was performed on the NextSeq500 system (Illumina). Pathological mutations were verified according to the classification standard of the American College of Medical Genetics and Genomics (ACMG) for germline mutations and the American Society of Clinical Oncology (ASCO)/College of American Pathologists (CAP) for somatic mutations. Mutation detection results were then used to select appropriate mutation-directed therapies for subsequent testing in the mini-PDX drug selection system. Microsatellite instability (MSI) detection was also included in this panel by testing $M L H 1, M S H 2, M S H 6$, and PMS2 genes. If high levels of MSI were detected (MSI-H), then immune checkpoint-inhibitor therapy was selected for patient administration. In addition to the 81 genes, we also analyzed single-nucleotide polymorphisms (SNPs) of 33 genes related to chemotherapeutic effects/side effects.

\section{The Establishment of Mini-PDX Models}

First, we acquired samples of tumor tissue. If patients had malignant pleural effusion and ascites, then we collected at least $500 \mathrm{~mL}$ of fluids in order to harvest tumor cells. Puncture biopsy was used to obtain tumor tissue samples from patients with certain conditions, such as liver metastases. If patients met the criteria for secondary tumorreduction surgery, then tissue samples were obtained during the surgery as long as this did not affect pathological diagnostic procedures.

We chose 4-6 types of chemotherapy regimens for mini-PDX drug-sensitivity tests according to the following principles: frequently used second-line chemotherapeutic plans for ovarian cancer (such as docetaxel, etoposide, and weekly paclitaxel) were commonly considered; unsatisfactory plans, as proven by medical history were excluded; and potentially effective molecular-targeted drugs/immunotherapeutic drugs, as indicated by mutation detection results were also selected. Drugs that might cause severe side effects, as indicated by medical history and genetic testing results, were avoided. Drug-sensitivity tests were carried out using mini-PDX models, as described later. Finally, we formulated a personalized chemotherapy plan for each patient according to the drug-sensitivity test results derived from the mini-PDX models.

\section{Immunofluorescence Studies}

Cytospins were prepared with cellularized tumor cells for immunohistochemical studies, as described previously. ${ }^{28}$ Then, cells were fixed with 4\% paraformaldehyde for 20 min, permeabilized with $0.3 \%$ Triton X-100 in PBS for 30 min, and blocked with 5\% normal goat serum for $1 \mathrm{~h}$ at room temperature. The cytospins were then incubated at $4^{\circ} \mathrm{C}$ overnight with primary rabbit/mouse monoclonal antibodies generated against the following proteins: WT1, a protein that is expressed at high levels in patients with epithelial ovarian cancer (1:600, ab89901, Abcam, Cambridge, UK); MUC16, a transmembrane glycoprotein expressed at high levels in over $80 \%$ of patients with ovarian cancer; CA125 (1:2000, ab110640, Abcam); and Ki67, as an indicator of tumor proliferation (1:400, ab245113, Abcam). Subsequently, a goat anti-rabbit secondary antibody (ab236466, Abcam) was applied for $10 \mathrm{~min}$ at room temperature; a mouse-specific reagent was incubated for $10 \mathrm{~min}$ prior to this step if a mouse primary antibody had been applied. Immunoreactive signals were visualized by incubation in diaminobenzidine (DAB) for 5 min. Finally, we captured images with a microscope (Leica DMI3000 B, Germany) and edited them with Photoshop (Adobe, USA).

\section{Mini-PDX Drug-Sensitivity Analysis}

Drug sensitivity was examined using the OncoVee ${ }^{\mathrm{TM}}-\mathrm{Mini}$ PDX assay (LIDE Biotech, Shanghai, China). This assay has been proven to be practical and exhibit a high success rate. ${ }^{28}$ In brief, ovarian carcinoma samples were washed with Hank's balanced salt solution (HBSS) to remove non-tumorous tissues and necrotic tumor tissues. After fragmentation, the tumor tissues were digested with collagenase at $37^{\circ} \mathrm{C}$ for 1-2 h. Once cells had been collected, we removed blood cells and fibroblasts and then transferred the resulting suspension of ovarian carcinoma cells to HBSS-washed capsules. Fourweek-old BALB/c nude mice (SLARC Inc., Shanghai, China), weighing 15-20 g each, were used for subcutaneous implantation. A small incision was made in the skin and the capsule was embedded in the subcutaneous tissue; each mouse received 4-6 capsules. Drugs were administered for seven consecutive days; normal saline was used as a control. We assessed antitumor activity based upon relative fluorescence units (RFUs) using the CellTiter-Glo ${ }^{\circledR}$ Luminescent Cell Viability Assay (Promega, Madison, WI, USA), and measured luminescence in terms of relative light units (RLU) using a spectrophotometer (SpectraMax M3, Molecular Devices, Sunnyvale, CA, USA). Tumor cell 
growth $(\mathrm{T} / \mathrm{C} \%=$ treatment group proliferation rate/control group proliferation rate \%) was calculated using the following formula: (mean RLU of the treatment group on day 7 mean RLU on day 0$) /($ mean RLU of the vehicle group on day 7 - mean RLU on day 0). The drug response in the mini-PDX system was represented by $(1-\mathrm{T} / \mathrm{C} \%)$; the lower the $\mathrm{T} / \mathrm{C} \%$, the greater the inhibitory effects of the regimen on the tumor. Generally, each mouse received 4-6 capsules, and each regimen was tested on two mice; the final results were calculated as the average of these six repeated trials. The protocol for the establishment of mini-PDX models is shown in Figure 1. All of the procedures were performed in specific pathogenfree conditions and were conducted in accordance with the guidelines of the Care and Use of Laboratory Animals of the National Institutes of Health, USA.

\section{Evaluation of Clinical Efficacy and Side- Effects}

The clinical effects of the recommended chemotherapy plan were evaluated by the Response Evaluation Criteria in Solid Tumors Version 1.1 (RECIST1.1) standards, ${ }^{30}$ in due consideration of the extent of tumor marker reduction, and the observed decrease in the size of the targeted lesions. Several markers are commonly used to evaluate tumors in patients with ovarian cancer, including CA125, CA199, CA153, HE4, CA74-2, CEA, and AFP. In the present study, we used the rate of reduction in CA125 expression as an indicator for anti-cancer effects since all patients exhibited a significant increase in their CA125 levels when recurrence occurred. Reductions in the size of the targeted lesions were evaluated by comparing tumor volume or maximal tumor diameter using ultrasonographic or magnetic resonance imaging (MRI) measurements. The degree of side effects was evaluated according to the Common Terminology Criteria for Adverse Events Version 5.0 (CTCAE v5.0) standards for ovarian cancer. Our primary endpoint was the clinical-benefit rate (the proportion of patients who underwent complete remission in addition to those with partial remission and those with

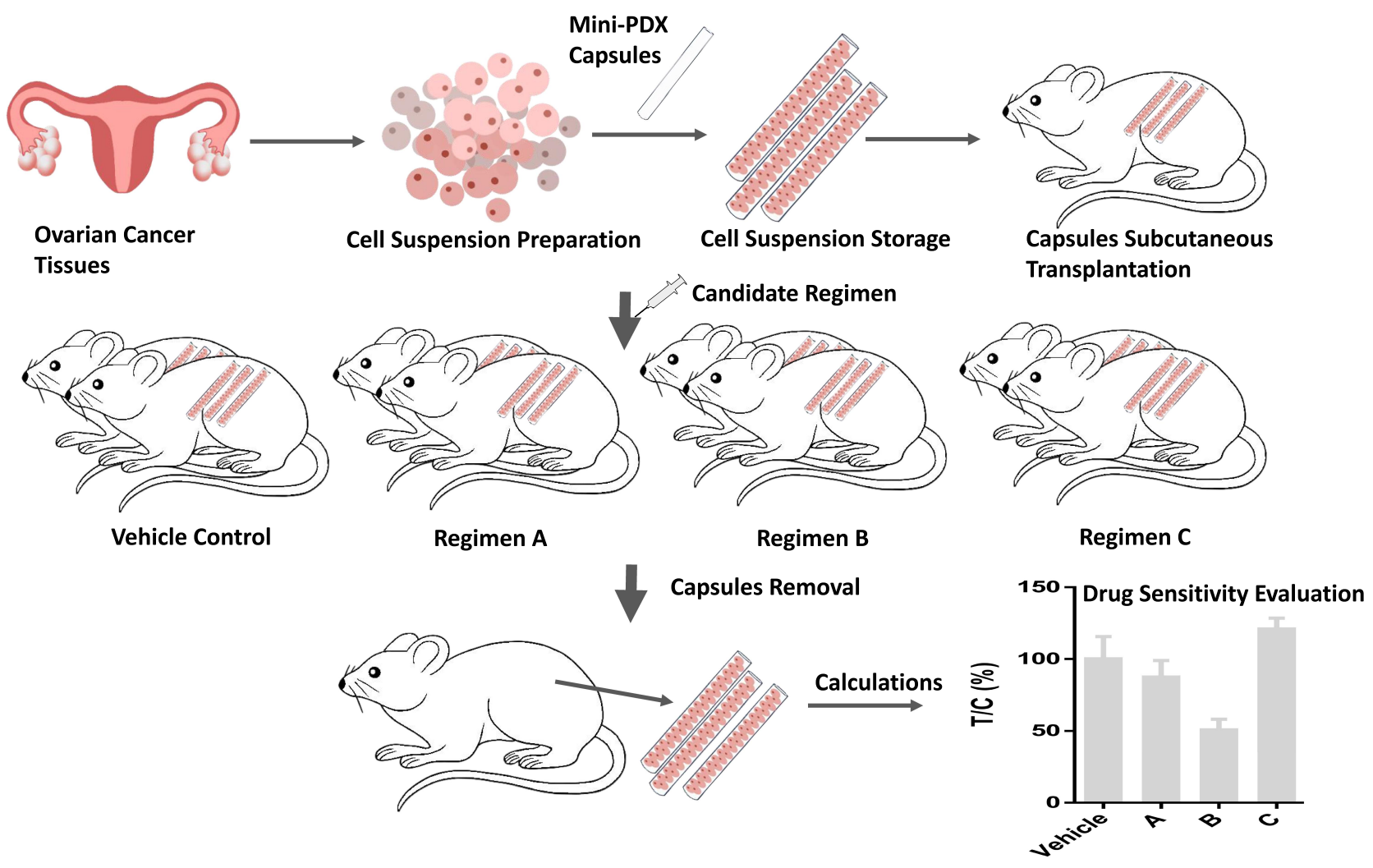

Figure I The protocol for establishing the mini-PDX models. Ovarian cancer cells were collected and transferred to capsules that had been washed in HBSS (Hank's Balanced Salt Solution). The capsules were subsequently implanted subcutaneously into BALB/c nude mice. Four to six chemotherapy regimens (and normal saline as a control) were then used to treat the mice. After 7 days of treatment, the capsules were removed and analyzed by the OncoVee ${ }^{\circledR}$ MiniPDX Assay for the rapid systemic detection of drug sensitivity. 
stable disease). We determined the number of severe side-effects (grade III or grade IV side-effects according to the CTCAEv5.0 standards) in order to evaluate the safety of our system.

\section{The Prediction of Side-Effects}

Potential side-effects for each regimen were investigated by determining the weight loss of mice in the mini-PDX system. Weight loss was recorded as RCBW\% (rate of change in body weight); this calculated as follows: $\mathrm{RCBW} \%=(\mathrm{BWi}-\mathrm{BW} 0) / \mathrm{BW} 0 * 100 \%$ in which $\mathrm{BWi}$ represented the body weight of the mouse on day 1 and BW0 represented the body weight of the mouse when the mini-PDX model began. Each regimen was tested on two mice; final RCBW\% was calculated as the average number of the two repeats; $15 \%$ was chosen as a cut-off point to predict whether we would consider avoiding this regimen due to the possibility of severe side-effects.

\section{Statistical Analysis}

The data-monitoring committee featured several members of the research group. The members of this committee ensured that patient privacy was maintained, registered patient information and updated the data in real time. Data are presented as means \pm standard deviation (SD).

\section{Ethical Statement}

Our study was approved by the Ethics Committee of the Obstetrics \& Gynecology Hospital of Fudan University (application number: 2016-51-X1) and all patients signed informed consent forms. The authors are willing to share the clinical data with all researchers upon request. Clinical trial data (including age, medical history, pathological examinations, imaging examination results, mutation detection results, mini-PDX drug-sensitivity results, and follow-up results) will be uploaded to ResMan (www.medresman.org) following publication of this article.

\section{Results}

\section{Basic Clinical Information}

Between January 2018 and December 2019, we enrolled 12 patients in to this study (Table 1). Mini-PDX models were successfully established using tumor fragments from each patient. The median duration of follow-up for the data analysis (data cut-off time was the March 30,2020 ) was 6.5 months. The mean age of the 12 patients was $52.75 \pm 7.01$ years. Eleven of the patients had ovarian high-grade serous adenocarcinoma; the remaining patient had ovarian clear cell carcinoma. Seven patients had platinum-resistant ovarian cancer in the conventional sense (a recurrence time within 6 months) and four patients had a recurrence more than 6 months after their last chemotherapy session; these latter four patients showed resistance to the carboplatin + paclitaxel (CP) regimen. Remarkably, patient 8 had been enrolled due to primary resistance to the $\mathrm{CP}$ regimen. One patient had stage-II disease (8.33\%); the others had advanced tumors (stages III-IV) at diagnosis. Most of the recurrent lesions were located in the pelvic cavity, accounting for $83.33 \%$ (10 patients). The remaining two patients exhibited their largest recurrent lesions in their liver/pelvic lymph nodes. The median size of recurrent tumors was $2.2 \mathrm{~cm}$; the largest tumor (patient 2) was $10.0 \mathrm{~cm}$. All of the patients showed a significant increase in CA125 levels when they were diagnosed with recurrent ovarian cancer; therefore CA125 levels were used to evaluate the clinical effects of treatment. More detailed clinical information regarding the 12 patients is shown in Table 1.

\section{Medical History}

On average, the patients had developed resistance to three categories of chemotherapeutic regimens prior to enrollment; one patient had failed five different types of chemotherapeutic regimens. Half of the patients had previously tried molecular-targeted drugs without the detection of any mutations, including bevacizumab, apatinib, and olaparib. The detailed medical history of the 12 patients is described in Table 2 .

\section{ctDNA Results}

The genetic-testing results showed that most of the 12 patients had tumor-related gene mutations. However, only a few of the patients had verified pathological mutations according to the classification standards recommended by the ACMG. Three patients had pathological BRCA mutations; all of these patients had a family history of tumors. A total of seven patients had molecular-targeted drugs that matched their gene mutations (including drugs in ongoing clinical trials). These same drugs were later selected for mini-PDX models. MSI-H was detected in only one of the 12 
Table I Baseline Patient Characteristics

\begin{tabular}{|c|c|c|c|c|c|c|}
\hline Number & Age & $\begin{array}{l}\text { Tumor } \\
\text { Staging }\end{array}$ & Pathological Types & Sites of Recurrence & $\begin{array}{l}\text { CAI } 25 \\
(\mathrm{U} / \mathrm{mL})\end{array}$ & $\begin{array}{c}\text { Regimen of Last } \\
\text { Chemotherapy }\end{array}$ \\
\hline I & 49 & IIIC & $\begin{array}{l}\text { High-grade serous } \\
\text { carcinoma }\end{array}$ & Pelvic cavity & $>5000$ & Non-platinum-based \\
\hline 2 & 51 & IIIC & $\begin{array}{l}\text { High-grade serous } \\
\text { carcinoma }\end{array}$ & Pelvic cavity & 251 & Platinum-based \\
\hline 3 & 62 & IV & $\begin{array}{l}\text { High-grade serous } \\
\text { carcinoma }\end{array}$ & $\begin{array}{c}\text { Pelvic cavity, liver } \\
\text { lesions }\end{array}$ & 488 & Non-platinum-based \\
\hline 4 & 62 & IV & $\begin{array}{l}\text { High-grade serous } \\
\text { carcinoma }\end{array}$ & Lymph nodes & $>5000$ & Platinum-based \\
\hline 5 & 51 & IIIC & $\begin{array}{l}\text { High-grade serous } \\
\text { carcinoma }\end{array}$ & Pelvic cavity & 321 & Platinum-based \\
\hline 6 & 43 & IV & $\begin{array}{l}\text { High-grade serous } \\
\text { carcinoma }\end{array}$ & Liver lesions & 128 & Non-platinum-based \\
\hline 7 & 54 & IIIC & $\begin{array}{l}\text { High-grade serous } \\
\text { carcinoma }\end{array}$ & Pelvic cavity & 215 & Non-platinum-based \\
\hline 8 & 54 & IIA & $\begin{array}{l}\text { High-grade serous } \\
\text { carcinoma }\end{array}$ & Pelvic cavity & 1526 & Non-platinum-based \\
\hline 9 & 56 & IIIC & $\begin{array}{l}\text { High-grade serous } \\
\text { carcinoma }\end{array}$ & Pelvic cavity & 146 & Non-platinum-based \\
\hline 10 & 63 & IIIC & $\begin{array}{l}\text { High-grade serous } \\
\text { carcinoma }\end{array}$ & Malignant ascites & 510 & Platinum-based \\
\hline 11 & 48 & IIIA & Clear cell carcinoma & Pelvic cavity & 348 & Platinum-based \\
\hline 12 & 40 & IIIC & $\begin{array}{l}\text { High-grade serous } \\
\text { carcinoma }\end{array}$ & Pelvic cavity & 364 & Non-platinum-based \\
\hline
\end{tabular}

Notes: Tumors were staged according to NCCN guidelines for ovarian cancer (2020-03). Sites of recurrence were defined by the location of the main recurrent lesions.

patients, indicating that immune checkpoint-inhibitor therapy might be an effective for of treatment. Drugmetabolizing enzyme SNPs showed which drugs might cause severe side effects. These findings, plus grade-I evidence according to the Clinical Pharmacogenetics Implementation Consortium (CPIC) guidelines were recorded. CtDNA results are shown in Table 3.

\section{Mini-PDX Drug-Sensitivity Results}

Sensitive regimens were identified for every patient, except for patient 10 (whose mini-PDX models showed resistance to all types of chemotherapeutic drug candidates). Our immunohistochemical study of luciferized tumor tissues in the mini-PDX models showed that the expression levels of MUC16, WT1, and Ki67 were consistent with epithelial ovarian cancer (Figure 2). Figure 3 depicts drug-sensitivity analysis (as $\mathrm{T} / \mathrm{C} \%$ ) while a detailed description of mini-PDX drug sensitivity results is given in Table 4, weight changes in each of the mouse models. A regimen was defined as "sensitive" when the proliferation rate of the tumor was under $50 \%$ compared to the control group. A mean of $1.75( \pm 1.01)$ different types of regimens showed satisfactory inhibitory effects $(\mathrm{T} / \mathrm{C} \%$ $<50 \%$ ) for each patient. The subsequent choice of chemotherapeutic regimen was guided by the drug-sensitivity results.

\section{Treatment Outcomes}

All of our patients used sensitive regimens which were predicted by the mini-PDX models except for patient 10. Following treatment, patient 6 (accounting for $8.33 \%$ of the total patient numbers) reached $\mathrm{CR}$ according to the RECIST1.1 standards. Patients 2, 3, 5, 9, and 12 reached PR (accounting for 41.66\%). In 
Table 2 Medical History of the Patients Included in This Study

\begin{tabular}{|c|c|c|c|c|c|c|}
\hline Number & $\begin{array}{l}\text { Primary } \\
\text { Onset }\end{array}$ & Primary Surgery & $\begin{array}{c}\text { Primary } \\
\text { Chemotherapy } \\
\text { History }\end{array}$ & $\begin{array}{l}\text { Recurrence } \\
\text { Recorded }\end{array}$ & $\begin{array}{l}\text { Type of } \\
\text { Platinum- } \\
\text { Resistance }\end{array}$ & $\begin{array}{c}\text { Treatment Before Enrollment (of } \\
\text { This Recurrence) }\end{array}$ \\
\hline I & $2016-02$ & $\begin{array}{l}2016-02 \\
\text { Cytoreduction (R0) }\end{array}$ & $\begin{array}{c}\text { TC*6, CR } \\
\text { Docetaxel: } 120 \\
\mathrm{mg}, \mathrm{C}: 650 \mathrm{mg}\end{array}$ & $2018-06$ & B & $\begin{array}{c}\mathrm{TC} * 2 \rightarrow \mathrm{G} * \mathrm{I} \\
\text { (Docetaxel: } 120 \mathrm{mg}, \mathrm{C}: 650 \mathrm{mg} \text { ) } \\
\text { G: } 1500 \mathrm{mg} \\
\text { (Gemcitabine } 1200 \mathrm{mg} \text { ) }\end{array}$ \\
\hline 2 & $2016-02$ & $\begin{array}{l}2016-02 \\
\text { Cytoreduction (R0) }\end{array}$ & $\begin{array}{c}\text { TC*6, CR } \\
\text { Docetaxel: } 120 \\
\mathrm{mg}, \mathrm{C}: 600 \mathrm{mg}\end{array}$ & $\begin{array}{l}2018-07 \\
2018-12\end{array}$ & A & 2018-12-26 Secondary cytoreduction (R0) \\
\hline 3 & $2015-04$ & $\begin{array}{l}\text { 20I5-04 } \\
\text { Cytoreduction (R0) }\end{array}$ & $\begin{array}{c}\text { TC*4, CR } \\
\text { Docetaxel: } 120 \\
\mathrm{mg}, \mathrm{C}: 600 \mathrm{mg}\end{array}$ & $\begin{array}{l}2017-01 \\
2018-04 \\
2018-09\end{array}$ & A & $\begin{array}{c}\text { TC*5(Docetaxel: } 120 \mathrm{mg}, \mathrm{C}: 650 \mathrm{mg} \text { ) } \\
\rightarrow \text { Olaparib*3 months } \\
\text { (Olaparib: } 300 \mathrm{mg} \text { bid PO.) }\end{array}$ \\
\hline 4 & $2017-10$ & $\begin{array}{l}\text { 20I7-II } \\
\text { Cytoreduction (R2) }\end{array}$ & $\begin{array}{c}\text { TC*6, CR } \\
\text { Docetaxel: } 120 \\
\mathrm{mg}, \mathrm{C}: 600 \mathrm{mg}\end{array}$ & $\begin{array}{l}2018-10 \\
2019-02\end{array}$ & A & $\begin{array}{c}\text { Cisplatin injection: } 40 \mathrm{mg} \text { pleural } \\
\text { perfusion*2 (this patient had malignant } \\
\text { pleural fluid) }\end{array}$ \\
\hline 5 & $2016-08$ & $\begin{array}{l}\text { 2016-08 } \\
\text { Cytoreduction (R0) }\end{array}$ & $\begin{array}{c}\text { TC*6,CR } \\
\text { Paclitaxel: } 210 \mathrm{mg} \\
\text { C: } 600 \mathrm{mg}\end{array}$ & $\begin{array}{l}2017-02 \\
2018-07\end{array}$ & A & $\begin{array}{l}\text { Paclitaxel: } 150 \mathrm{mg} \text { weekly*4 } \rightarrow \\
\text { TC*5 (Docetaxel: } 120 \mathrm{mg}, \mathrm{C}: 600 \mathrm{mg}) \rightarrow \\
\text { Secondary Cytoreduction (R0) }\end{array}$ \\
\hline 6 & $2015-08$ & $\begin{array}{l}\text { 20I5-08 } \\
\text { Cytoreduction (R0) }\end{array}$ & $\begin{array}{c}\text { TC*8 } \\
\text { Paclitaxel: } 210 \\
\mathrm{mg}, \mathrm{C}: 600 \mathrm{mg}\end{array}$ & $\begin{array}{l}2017-02 \\
2018-04\end{array}$ & A & $\begin{array}{l}\text { Liposomal Dox+Nedaplatin*3 (Day I: } \\
\text { Liposomal Dox } 20 \text { mg Day I-Day 2: } \\
\text { Nedaplatin } 50 \text { mg) } \rightarrow \text { Apatinib+Olaparib } \\
\text { maintenance treatment for I year } \\
\text { (Olaparib: } 300 \text { mg bid PO. Apatinib: } 750 \\
\text { mg qd PO.) }\end{array}$ \\
\hline 7 & $2018-05$ & $\begin{array}{l}\text { 2018-07 } \\
\text { Cytoreduction (R0) }\end{array}$ & $\begin{array}{c}\text { TC*5, CR } \\
\text { Docetaxel: } 120 \\
\mathrm{mg}, \mathrm{C}: 600 \mathrm{mg}\end{array}$ & $2019-06$ & B & $\begin{array}{l}\text { TC*3 (Day I: Paclitaxel } 210 \text { mg+Day } 2 \text { : } \\
\text { Lobaplatin } 40 \mathrm{mg}) \rightarrow \text { Secondary } \\
\text { cytoreduction }(\mathrm{R} 0) \rightarrow \mathrm{G} * 3(\text { Gemcitabine: } \\
\text { I } 200 \mathrm{mg})\end{array}$ \\
\hline 8 & $2018-04$ & $\begin{array}{l}\text { 2018-05 } \\
\text { Cytoreduction (R0) }\end{array}$ & $\begin{array}{l}\text { Primary } \\
\text { treatment result: } \\
\text { PD }\end{array}$ & I & C & $\begin{array}{c}\text { TC*3 (Day I: Paclitaxel } 210 \mathrm{mg}+\text { Day I-3: } \\
\text { Cisplatin } 30 \mathrm{mg} \text { ) } \rightarrow \text { Gemcitabine (Day } \\
\text { I-3:1400 mg)*2 } \rightarrow \text { Paclitaxel+ } \\
\text { Bevacizumab*3 (Day I: Paclitaxel } 90 \mathrm{mg} \\
\text { +Bevacizumab } 400 \mathrm{mg} \text {, Day 8+Day I5: } \\
\text { Paclitaxel } 90 \mathrm{mg} \text { ) }\end{array}$ \\
\hline 9 & $2016-10$ & $\begin{array}{l}2016-10 \\
\text { Cytoreduction (R0) }\end{array}$ & $\begin{array}{c}\text { TC*8, CR } \\
\text { Docetaxel: } 120 \\
\mathrm{mg}, \mathrm{C}: 600 \mathrm{mg}\end{array}$ & $\begin{array}{l}2018-01 \\
2018-12 \\
2019-10\end{array}$ & A & $\begin{array}{l}\text { Etoposide (Etoposide } 50 \mathrm{mg} \text { qd PO.)*20 } \\
\text { days } \rightarrow \text { Bevacizumab } 500 \mathrm{mg} \text { once }\end{array}$ \\
\hline 10 & $2015-10$ & $\begin{array}{l}\text { 20I5-II } \\
\text { Cytoreduction (R0) }\end{array}$ & $\begin{array}{c}\text { TC*6 } \\
\text { Paclitaxel: } 210 \\
\mathrm{mg}, \mathrm{C}: 600 \mathrm{mg}\end{array}$ & $\begin{array}{l}2017-08 \\
2018-06 \\
2019-04 \\
\text { (recurrence } \\
\quad \text { during } \\
\text { Olaparib } \\
\text { treatment) }\end{array}$ & A & $\begin{array}{c}\text { Olaparib*4 months } \\
\text { (Olaparib: } 300 \mathrm{mg} \text { bid PO.) } \rightarrow \text { Paclitaxel+ } \\
\text { Bevacizumab*4 (Day I: Paclitaxel I } 20 \mathrm{mg} \\
\text { +Bevacizumab } 400 \mathrm{mg} \text {, Day 8+Day I5: } \\
\text { Paclitaxel I } 20 \mathrm{mg} \text { ) } \rightarrow \text { Topotecan (Day I, 8, and } \\
\text { 15: Topotecan } 4 \mathrm{mg} \text { ) } \rightarrow \text { Pemetrexed } 800 \mathrm{mg} \\
\text { +Cisplatin } 100 \mathrm{mg} \text { once }\end{array}$ \\
\hline
\end{tabular}

(Continued) 
Table 2 (Continued).

\begin{tabular}{|c|c|c|c|c|c|c|}
\hline Number & $\begin{array}{l}\text { Primary } \\
\text { Onset }\end{array}$ & Primary Surgery & $\begin{array}{c}\text { Primary } \\
\text { Chemotherapy } \\
\text { History }\end{array}$ & $\begin{array}{l}\text { Recurrence } \\
\text { Recorded }\end{array}$ & $\begin{array}{c}\text { Type of } \\
\text { Platinum- } \\
\text { Resistance }\end{array}$ & $\begin{array}{c}\text { Treatment Before Enrollment (of } \\
\text { This Recurrence) }\end{array}$ \\
\hline II & $2018-06$ & $\begin{array}{l}2018-06 \\
\text { Cytoreduction (R0) }\end{array}$ & $\begin{array}{c}\text { TC*6, CR } \\
\text { Docetaxel: } 90 \mathrm{mg} \text {, } \\
\text { C: } 500 \mathrm{mg}\end{array}$ & $2019-05$ & A & $\begin{array}{c}\text { Paclitaxel weekly treatment*3 (Day I, 8, } \\
\text { and I5: Paclitaxel } 90 \mathrm{mg} \text { ) } \rightarrow \text { Gemcitabine } \\
\text { +Oxaliplatin (Day I and 8: Gemcitabine } \\
\text { I200 mg, Day I: Oxaliplatin I } 20 \\
\text { mg) } \rightarrow \text { Irinotecan+Cisplatin*I (Day I: } \\
\text { Irinotecan } 70 \text { mg, Day I and 8: Cisplatin } 70 \\
\text { mg) }\end{array}$ \\
\hline 12 & $2013-11$ & $\begin{array}{l}2018-06 \\
\text { Cytoreduction (R0) }\end{array}$ & $\begin{array}{c}\text { TC*8 } \\
\text { Paclitaxel: } 210 \\
\mathrm{mg}, \mathrm{C}: 700 \mathrm{mg}\end{array}$ & $\begin{array}{l}2016-04 \\
2018-10\end{array}$ & B & $\begin{array}{c}\text { TC+Bev*4 (Day I: Paclitaxel 210 mg } \\
\text { +Bevacizumab } 400 \text { mg, Day 2: Oxaliplatin } \\
\text { I30 mg) } \rightarrow \text { Bevacizumab*4 (Day I: } \\
\text { Bevacizumab } 400 \mathrm{mg}) \rightarrow \text { Cytoreduction } \\
(\mathrm{RI})\end{array}$ \\
\hline
\end{tabular}

Notes: "*n" refers to the number of cycles of this regimen. R0: R0-section, section with no visible lesions. RI: RI-section, section with a lesion diameter $\leq$ Icm; R2: R2section, section with a lesion diameters $>I \mathrm{~cm}$. Treatment results of primary onset followed the RECISTI.I standard. Type of platinum-resistance: A. Conventional platinumresistant recurrence of ovarian cancer: the recurrence occurred within 6 months from the last platinum-based chemotherapy; B. The recurrence occurred after 6 months from the last chemotherapy, but the tumor was actually platinum-resistant; C. Primary ovarian cancer but resistant to platinum-based regimens. The latest recurrence recorded was when the patient was enrolled. If not mentioned otherwise, all drugs were used intravenously. The interval between each cycle was usually 3 weeks if not mentioned otherwise. Detailed doses are given as follows: TC: Day I Docetaxel injection/Paclitaxel injection+Day 2 Carboplatin injection(C); G: Day I+Day 8 Gemcitabine hydrochloride for injection.

addition, $25 \%$ of patients $(1,7$, and 11$)$ reached SD after treatment. However, patients 4,8 , and 10, all showed disease progression. The overall clinical-benefit rate was $75 \%$ (Figure 4). The detailed treatment plans that were established after drug selection, along with the clinical outcomes of each patient, are shown in Table 5. Specifically, although patient 1 reached SD after two cycles of liposomal doxorubicin followed by four cycles of docetaxel + carboplatin, her chief doctor then used another four cycles of albumin-bound paclitaxel (over 3 weeks) as consolidation therapy, and leaving the CA125 level and tumor size as before the consolidation therapy. Patient 4, who had MSI-H, used pembrolizumab for immune checkpoint-inhibitor treatment on one occasion; however, her disease progressed.

Table 3 CtDNA Results for the 12 Patients

\begin{tabular}{|c|c|c|c|c|c|}
\hline Number & $\begin{array}{c}\text { Pathological } \\
\text { Mutations }\end{array}$ & $\begin{array}{l}\text { Mutation } \\
\text { Frequency }\end{array}$ & $\begin{array}{l}\text { Mutation } \\
\text { Type }\end{array}$ & Matched Targeted Drug & $\begin{array}{c}\text { Microsatellite } \\
\text { Status }\end{array}$ \\
\hline 1 & None & l & & l & Unreported \\
\hline 2 & $B R C A I$ & $45.63 \%$ & Germline mutation & Olaparib & MSS \\
\hline 3 & TP53 & $9.43 \%$ & Somatic mutation & AZDI775 (clinical trial) & MSS \\
\hline 4 & TP53 & $6.78 \%$ & Somatic mutation & AZDI775 (clinical trial) & MSI-H \\
\hline 5 & None & l & & l & MSI-L \\
\hline 6 & MYC & Gene amplification & Somatic mutation & TAS-I I9, Roniciclib (clinical trial) & MSS \\
\hline 7 & None & I & & I & MSI-L \\
\hline 8 & None & l & & l & MSI-L \\
\hline 9 & None & l & & 1 & MSS \\
\hline 10 & BRCAI & $49 \%$ & Germline mutation & Olaparib & MSS \\
\hline \multirow[t]{2}{*}{ II } & ATM & $48.4 \%$ & Germline mutation & Olaparib & MSI-L \\
\hline & KRAS & $5.4 \%$ & Somatic mutation & Cobimetinib, Binimetinib, Trametinib & \\
\hline 12 & BRCAI & $49.5 \%$ & Germline mutation & Olaparib & MSI-L \\
\hline
\end{tabular}

Notes: Microsatellite status represents a measure of microsatellite stability. Results are reported as MSI-High (MSI-H), MSI-Low (MSI-L), and MSI-Stable (MSS). AZDI775 and some other targeted drugs were still involved in clinical trials and were not available in China at the time of this study; these particular drugs were not selected for miniPDX models. 


\section{Mini-PDX Model}
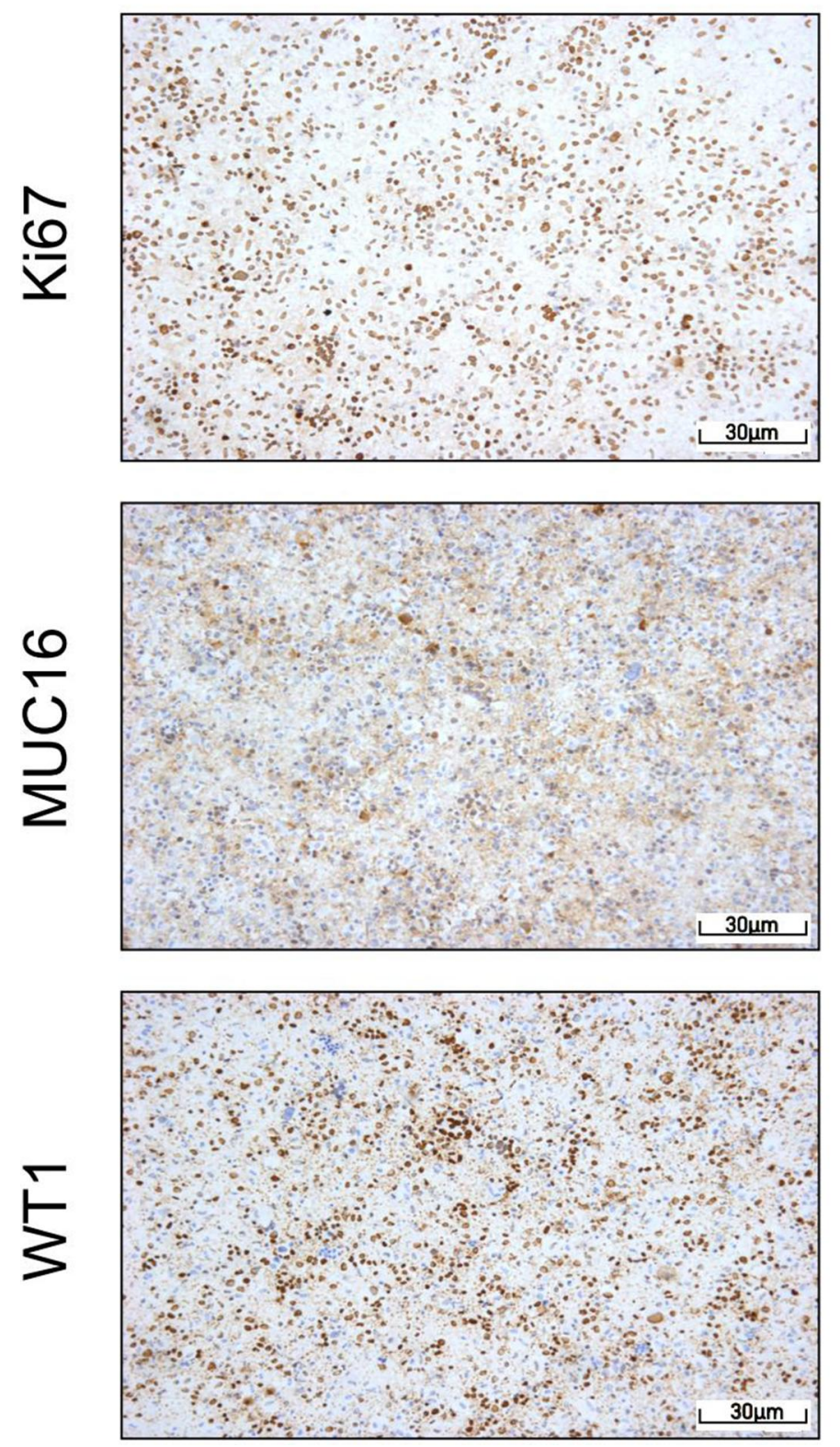

\section{Ovarian Cancer Tissues}
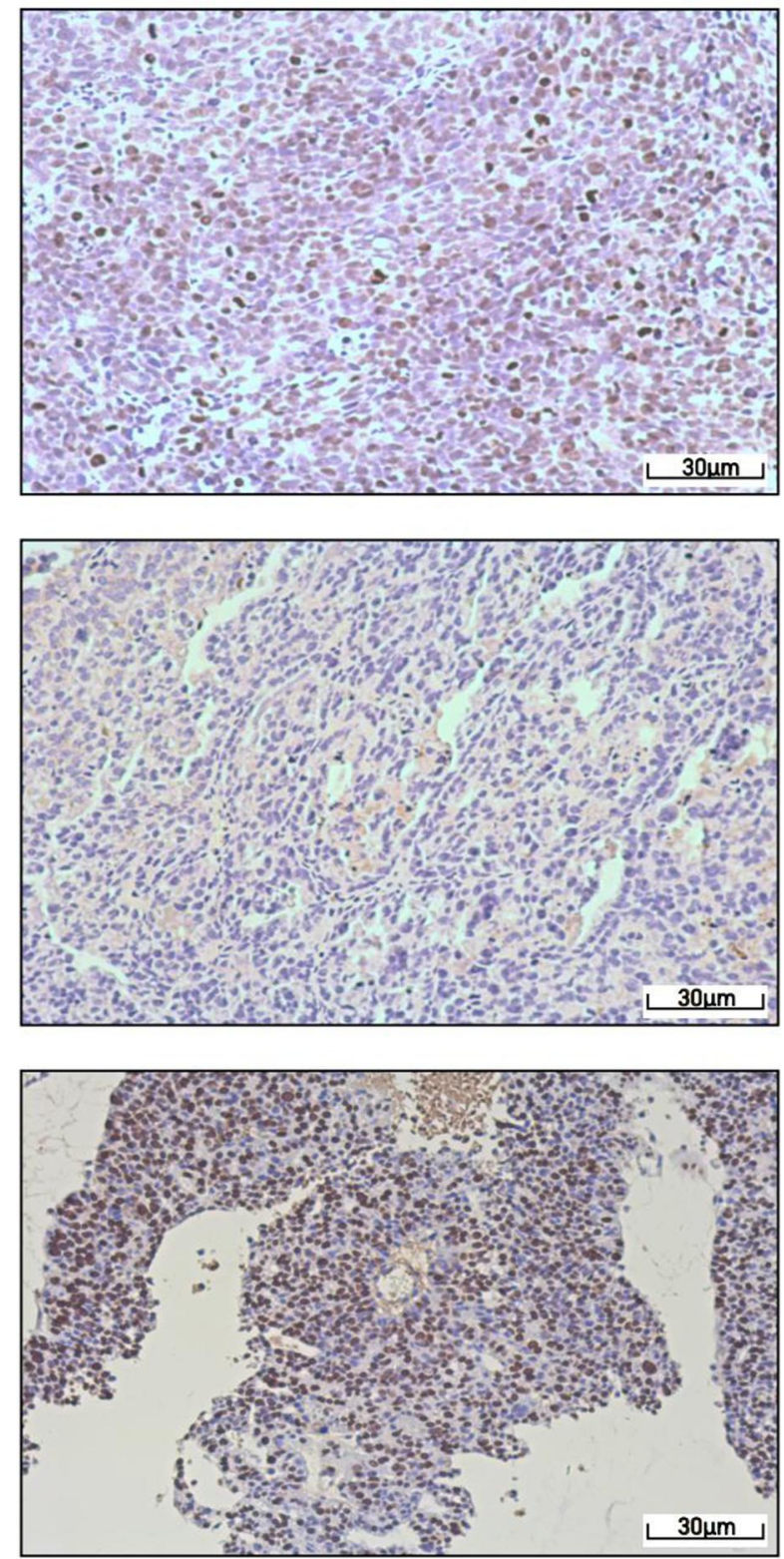

Figure 2 Mini-PDX models demonstrated that the expression levels of IHC markers were consistent with epithelial ovarian cancer tissues (pKi67, MUCI6, and WTI positive).

\section{Side Effect Predictions and Measurements}

If the ctDNA results predicted that a particular patient might suffer from severe side effects arising from the administration of one regimen according to drug-metabolizing enzyme SNPs, then this drug was excluded as a candidate for our mini-PDX models. Furthermore, if a mouse model suffered a loss in weight that exceeded
$15 \%$ during the 7-day treatment period, then we considered it possible that this regimen would also cause serious side effects. Alternatives were considered when the inhibitory rate was similar. The degree of weight loss for each mouse model is shown in Figure 5. We found that the nude mice matched to patients 2, 5, 9, and 10, showed notable weight loss 


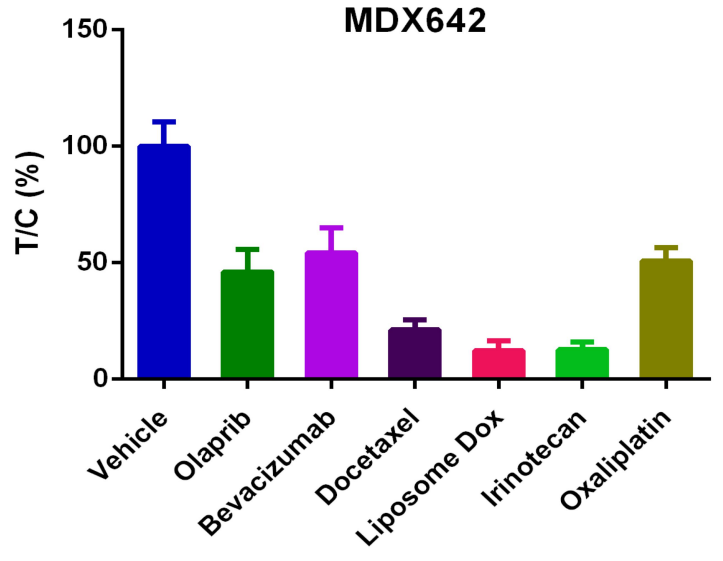

Patient No. 1

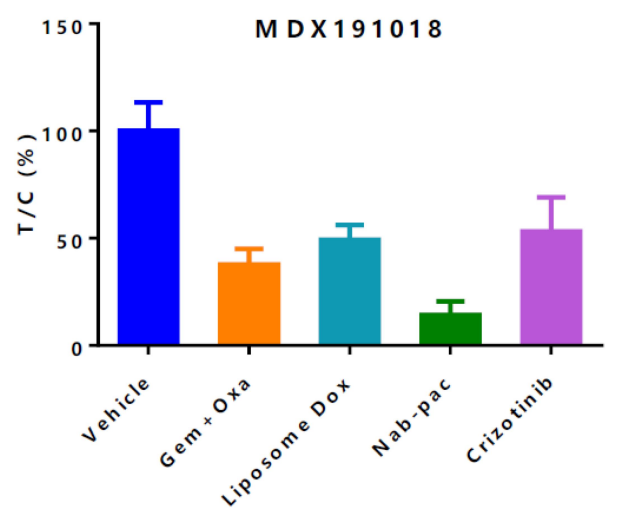

Patient No. 3

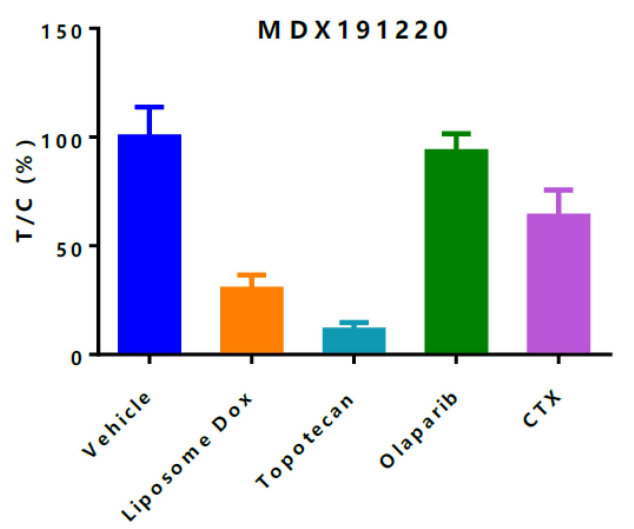

Patient No. 5

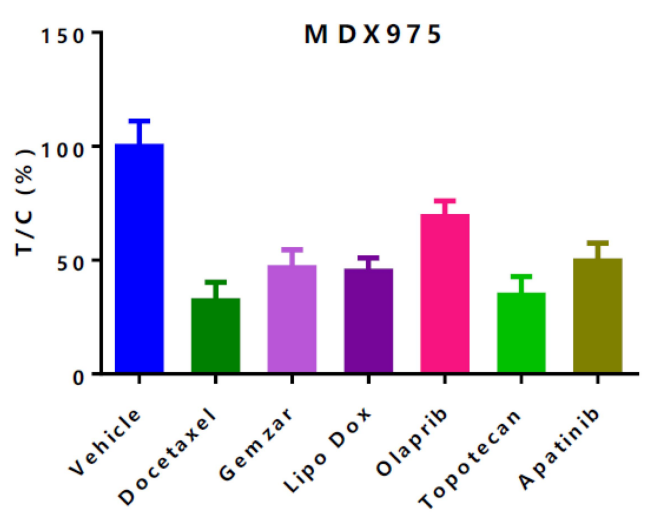

Patient No. 2

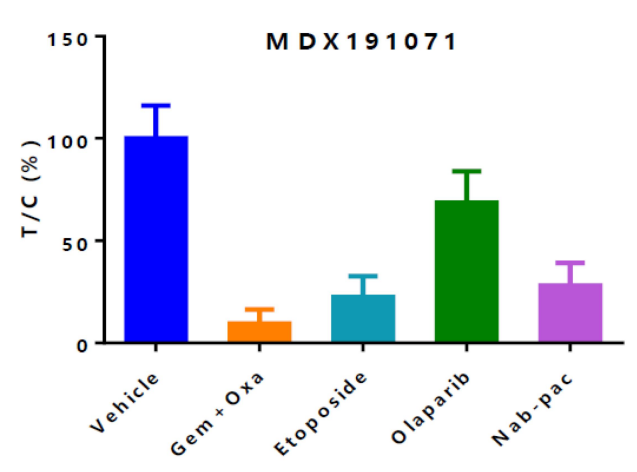

Patient No. 4

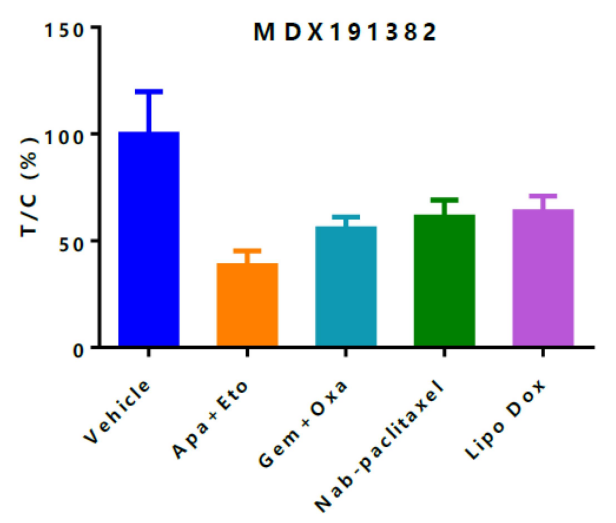

Patient No. 6

Figure 3 Continued. 


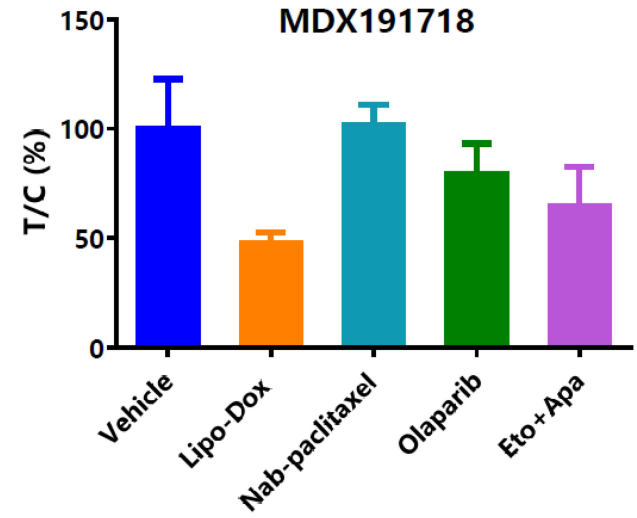

Patient No. 7

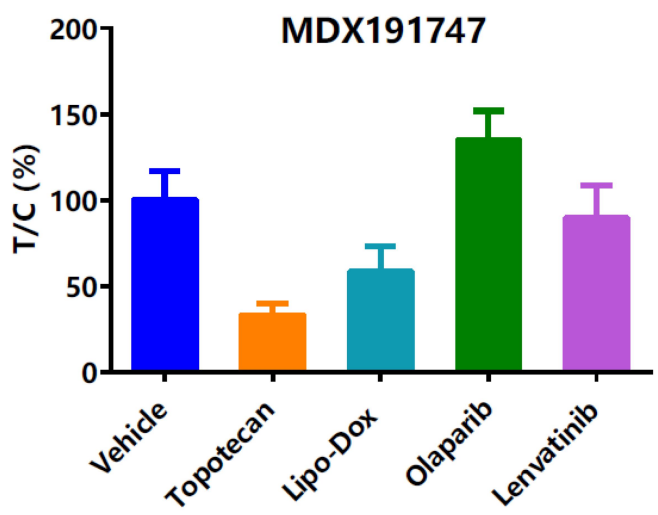

Patient No. 9

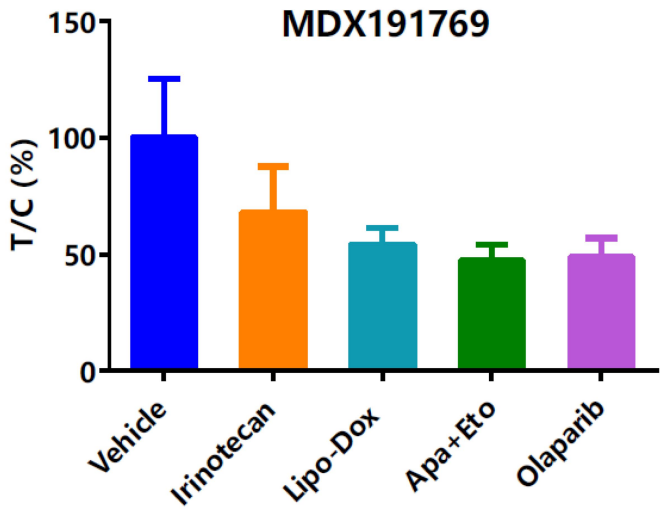

Patient No. 11

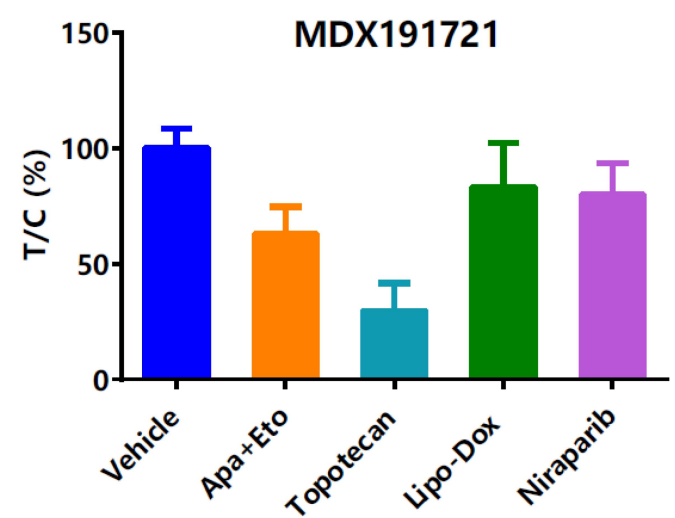

Patient No. 8

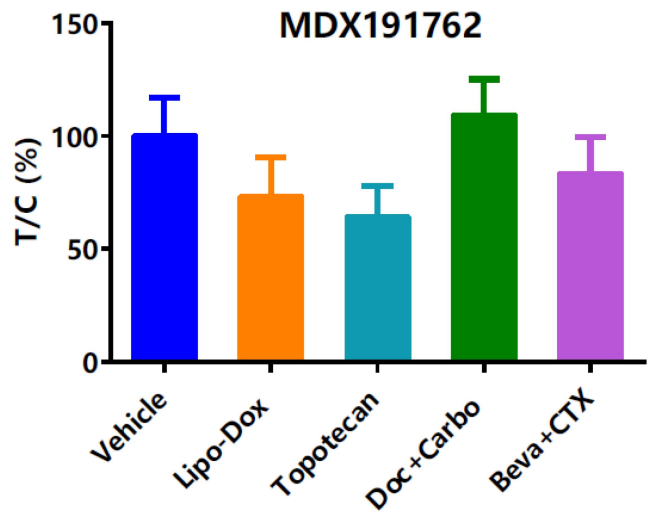

Patient No. 10

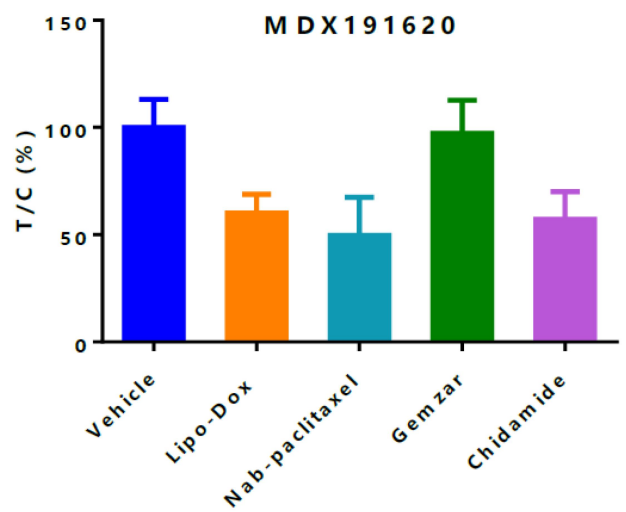

Patient No. 12

Figure 3 Drug-selection test results for the mini-PDX models based on the 12 patients. Tumor cell growth (T/C\% = treatment group proliferation rate/control group proliferation rate \%) was calculated using the formula: (mean RLU of the treatment group on day 7 - mean RLU on day 0)/(mean RLU of the vehicle group on day 7 - mean RLU on day 0 ). Abbreviations for chemotherapy: Liposomal doxorubicin (Lipo Dox); Nab-pac (Nab-paclitaxel); Gem+Oxa (Gemcitabine+Oxaliplatin); CTX (Cyclophosphamide); Apa+Eto (Apatinib+Etoposide); Doc +Carbo (Docetaxel+Carboplatin); Bev+CTX (Bevacizumab+Cyclophosphamide); Gemzar (Gemcitabine). 
Table 4 Mini-PDX Drug Sensitivity Results for the 12 Patients

\begin{tabular}{|c|c|c|c|c|}
\hline Number & Chemotherapy Regimen & Inhibition Rate (I-T/C\%) & Weight Loss of Mice $>15 \%$ & Source of Cancer Cells \\
\hline I & $\begin{array}{l}\text { I. Liposomal Dox } \\
\text { 2. Irinotecan } \\
\text { 3. Docetaxel } \\
\text { 4. Olaparib } \\
\text { 5. Oxaliplatin } \\
\text { 6. Bevacizumab }\end{array}$ & \begin{tabular}{l|}
$88 \%$ \\
$86 \%$ \\
$78 \%$ \\
$54 \%$ \\
$49 \%$ \\
$46 \%$
\end{tabular} & $\begin{array}{l}- \\
- \\
- \\
- \\
- \\
-\end{array}$ & Malignant ascites \\
\hline 2 & $\begin{array}{l}\text { I. Docetaxel } \\
\text { 2. Liposomal Dox } \\
\text { 3. Olaparib } \\
\text { 4. Topotecan } \\
\text { 5. Apatinib }\end{array}$ & $\begin{array}{l}68 \% \\
53 \% \\
55 \% \\
65 \% \\
50 \%\end{array}$ & $\begin{array}{l}- \\
- \\
- \\
+ \\
-\end{array}$ & Ovarian cancer tissues from surgery \\
\hline 3 & $\begin{array}{l}\text { I. Nab-paclitaxel } \\
\text { 2. Gemcitabine+Oxaliplatin } \\
\text { 3. Liposomal Dox } \\
\text { 4. Crizotinib }\end{array}$ & $\begin{array}{l}86 \% \\
62 \% \\
51 \% \\
47 \%\end{array}$ & $\begin{array}{l}- \\
- \\
+\end{array}$ & Liver metastases puncture biopsy tissues \\
\hline 4 & $\begin{array}{l}\text { I. Gemcitabine+Oxaliplatin } \\
\text { 2. Etoposide } \\
\text { 3. Nab-paclitaxel } \\
\text { 4. Olaparib }\end{array}$ & $\begin{array}{l}91 \% \\
77 \% \\
72 \% \\
41 \%\end{array}$ & $\begin{array}{l}- \\
- \\
-\end{array}$ & Malignant pleural fluid \\
\hline 5 & $\begin{array}{l}\text { I. Topotecan } \\
\text { 2. Liposomal Dox } \\
\text { 3. Cyclophosphamide } \\
\text { 4. Olaparib }\end{array}$ & $\begin{array}{c}89 \% \\
70 \% \\
46 \% \\
7 \%\end{array}$ & $\begin{array}{l}+ \\
- \\
- \\
-\end{array}$ & Ovarian cancer tissues from surgery \\
\hline 6 & $\begin{array}{l}\text { I. Apatinib+Etoposide } \\
\text { 2. Gemcitabine+Oxaliplatin } \\
\text { 3. Nab-paclitaxel } \\
\text { 4. Liposomal Dox }\end{array}$ & $\begin{array}{l}62 \% \\
45 \% \\
39 \% \\
36 \%\end{array}$ & $\begin{array}{l}- \\
- \\
-\end{array}$ & Liver metastases puncture biopsy tissues \\
\hline 7 & $\begin{array}{l}\text { I. Liposomal Dox } \\
\text { 2. Apatinib+Etoposide } \\
\text { 3. Olaparib } \\
\text { 4. Nab-paclitaxel }\end{array}$ & $\begin{array}{l}52 \% \\
36 \% \\
21 \% \\
-2 \%\end{array}$ & $\begin{array}{l}- \\
- \\
-\end{array}$ & Ovarian cancer tissues from surgery \\
\hline 8 & $\begin{array}{l}\text { I. Topotecan } \\
\text { 2. Apatinib+Etoposide } \\
\text { 3. Niraparib } \\
\text { 4. Liposomal Dox }\end{array}$ & $\begin{array}{l}70 \% \\
47 \% \\
20 \% \\
17 \%\end{array}$ & $\begin{array}{l}- \\
- \\
-\end{array}$ & Malignant ascites \\
\hline 9 & $\begin{array}{l}\text { I. Topotecan } \\
\text { 2. Liposomal Dox } \\
\text { 3. Lenvatinib } \\
\text { 4. Olaparib }\end{array}$ & $\begin{array}{r}67 \% \\
42 \% \\
10 \% \\
-35 \%\end{array}$ & $\begin{array}{l}+ \\
- \\
- \\
-\end{array}$ & Malignant ascites \\
\hline 10 & $\begin{array}{l}\text { I. Topotecan } \\
\text { 2. Liposomal Dox } \\
\text { 3. Cyclophosphamide+Bevacizumab } \\
\text { 4. Docetaxel+Carboplatin }\end{array}$ & $\begin{array}{l}36 \% \\
28 \% \\
17 \% \\
-9 \%\end{array}$ & $\begin{array}{l}- \\
- \\
- \\
+\end{array}$ & Malignant ascites \\
\hline 11 & $\begin{array}{l}\text { I. Apatinib+Etoposide } \\
\text { 2. Olaparib } \\
\text { 3. Liposomal Dox } \\
\text { 4. Irinotecan }\end{array}$ & $\begin{array}{l}53 \% \\
51 \% \\
46 \% \\
32 \%\end{array}$ & $\begin{array}{l}- \\
- \\
-\end{array}$ & Malignant ascites \\
\hline
\end{tabular}

(Continued) 
Table 4 (Continued).

\begin{tabular}{|l|l|c|l|l|}
\hline Number & Chemotherapy Regimen & Inhibition Rate (I-T/C\%) & Weight Loss of Mice> I5\% & Source of Cancer Cells \\
\hline \multirow{2}{*}{12} & I. Nab-paclitaxel & $50 \%$ & - & Ovarian cancer tissues from surgery \\
& 2. Chidamide & $43 \%$ & - & \\
& 3. Liposomal Dox & $40 \%$ & - & \\
& 4. Gemcitabine & $3 \%$ & - & \\
\hline
\end{tabular}

Notes: Inhibition rate was calculated by I-T/C\% (T/C\%=treatment group proliferation rate/control group proliferation rate\%). Each regimen was used on mini-PDX models with the same doses. Detailed doses for the mini-PDX models: Liposomal doxorubicin (Lipo-Dox): $5 \mathrm{mg} / \mathrm{kg}$, IP (intraperitoneal injection), on Day I and Day 5; Irinotecan: 50 $\mathrm{mg} / \mathrm{kg}$, IP, on Day I and Day 5; Docetaxel: $20 \mathrm{mg} / \mathrm{kg}$, IP, on Day I and Day 5; Olaparib: $100 \mathrm{mg} / \mathrm{kg}$, PO (oral administration), Day I-Day 7; Oxaliplatin: $5 \mathrm{mg} / \mathrm{kg}$, IP, on Day I and Day 5; Bevacizumab: 10 mg/kg, IP, on Day I and Day 5; Topotecan: 4 mg/kg, IP, Day I-Day 5; Apatinib: 100 mg/kg, PO, Day I-Day 7; Nab-paclitaxel (Nab-pac): 20 mg/kg, IV (caudal vein injection), Day I-Day 5; Gemcitabine+Oxaliplatin (Gem+Oxa): Gemcitabine, 60 mg/kg, IP, Day I and Day $5+$ Oxaliplatin 5 mg/kg, IP, Day I; Crizotinib: 50 mg/ kg, PO, Day I-Day 7; Etoposide: 20 mg/kg, IP, Day I and Day 5; Apatinib+Etoposide (Apa+Eto): Apatinib, I00 mg/kg, PO, Day I-Day 7 + Etoposide, 20 mg/kg, IP, Day I and Day 5; Docetaxel+Carboplatin: Docetaxel (Doc), $20 \mathrm{mg} / \mathrm{kg}$, IP, Day I and Day 5; Carboplatin (Carbo), $50 \mathrm{mg} / \mathrm{kg}$, IP, Day I; Irinotecan: $50 \mathrm{mg} / \mathrm{kg}$, IP, Day I and Day 5; Chidamide: 50 mg/kg, PO, Day I-7; Gemcitabine (Gemzar): 60 mg/kg, IP, Day I and Day 5; Cyclophosphamide (CTX): 100 mg/kg, IP, Day I and Day 5; Lenvatinib: I00 mg/kg, PO, Day I-7; Cyclophosphamide+Bevacizumab (CTX+Bev): Bevacizumab, 10 mg/kg, IP, Day I and Day $5+$ Cyclophosphamide, I00 mg/kg, IP, Day I and Day 5.

caused by topotecan. Patients 5 and 9 continued to use topotecan as their subsequent chemotherapeutic regimen due to its outstanding performance in the miniPDX models; patient 5 developed grade-III bone marrow depression. Milder side effects, according to the CTCAEv5.0 standards, are described in Table 5. Patient 5 was the only patient who showed serious side effects; her chemotherapeutic procedure was postponed for 1 week.

\section{Reductions in the CAI25 Tumor Marker}

Since all recurrences observed in the 12 patients were accompanied by significant increases in CA125 levels, we used the rate of decrease in this tumor marker to evaluate the anti-tumor effects of chosen regimens. We observed a significant decline (over 20\%) in the CA125 levels of seven patients after using the recommended regimen; the CA125 levels in two patients were augmented after treatment; these patients also showed disease progression. The remaining three patients exhibited stable CA125 levels; the median rate of decline in CA125 was $39.63 \%$. The changes in CA125 levels after treatment are shown in Figure 6.

\section{Discussion}

An increasing number of individualized therapies that are based on genetic-testing results are been applied to patients with ovarian cancer. However, most of the existing studies select corresponding targeted drugs or immunotherapy drugs for patients in accordance with the results of genetic testing. Even for patients with germline $B R C A$ mutations, olaparib treatment only showed an ORR (Overall Response Rate) of 34\%; this was not much higher than the ORRs of patients treated with common second-line chemotherapeutic drugs. $^{31-35}$ These findings indicate that genetic testing alone is not sufficient to achieve satisfactory effects. Therefore, it is clear that we are in desperate need of a more efficient and more direct drug-screening system that can provide accurate predictions of drug effects in real time and where the predictive results have a high level of consistency with clinical results. Treatments that follow a novel personalized drug-screening system can provide better outcomes for patients with platinum-resistant ovarian cancer when they exhibit advanced tumors that are refractory to standard treatment.

Fortunately, an increasing number of novel methods for precision medicine are now available. The I-PREDICT study proposed the new concept of a "matching score" with which to evaluate the degree to which a patient's genetic mutations could be matched to targeted drugs. ${ }^{36}$ Consequently, it was demonstrated that the more pathological genetic mutations could be matched, the better the prognosis that could be achieved. Moreover, functional diagnoses have become more significant since drug-screening tests using tumor cell lines do not truly reflect the clinical response of tumors in vivo. ${ }^{37,38}$

Our results indicated that the use of mini-PDX models as a personalized drug-screening system resulted in a 


\section{Distribution of treatment outcomes}

A

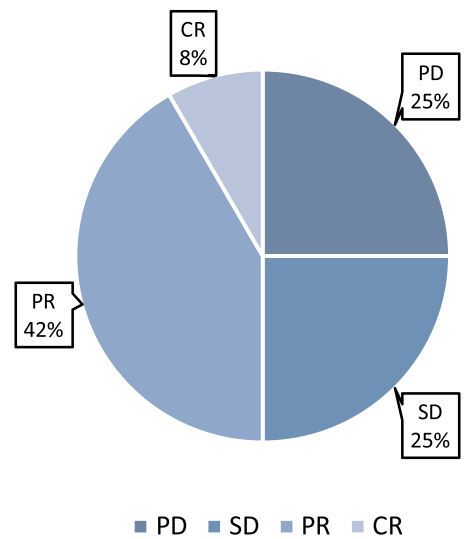

B

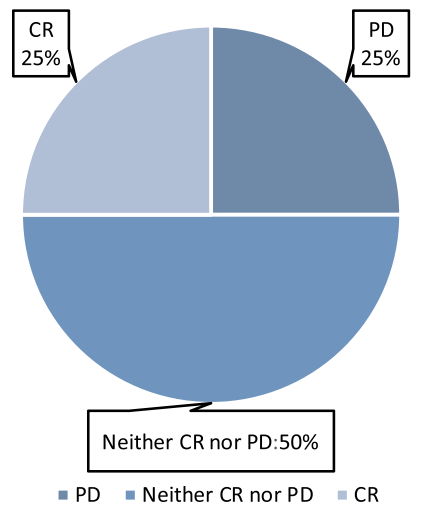

C

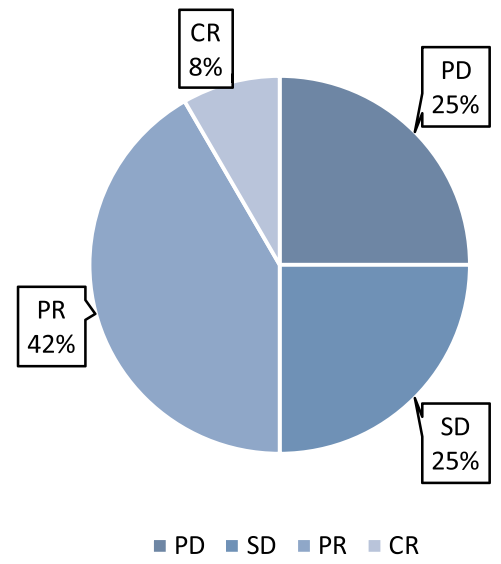

Figure 4 Distribution of treatment outcomes, including the treatment outcomes of targeted lesions (A), non-targeted lesions (B), and overall outcomes (C). The outcomes of treatment were defined according to the RECISTI.I standard. The overall clinical-benefit rate was $75 \%$.

Abbreviations: $\mathrm{CR}$, complete remission; PR, partial remission; SD, stable disease; $\mathrm{PD}$, progression of disease.

clinical benefit rate of $75 \%$ for patients with platinumresistant ovarian cancer; this was much higher than the mean clinical benefit rate of patients treated with a singleagent, second-line chemotherapy. With these data, it is reasonable for us to expect greater utilization of personalized drug-screening systems to treat platinum-resistant ovarian cancer, as well as more-refractory advanced tumors.

In addition, the mini-PDX system might change the way we think about the categorization of recurrent ovarian cancer. Using the present guidelines, if a recurrence occurs within 6 months of the last chemotherapy session, then this condition would be diagnosed with "platinum-resistant recurrence"; otherwise, the patient would be diagnosed with "platinum-sensitive recurrence" and the first choice of treatment would be platinum-based regimens. However, physicians have noted that some so-called platinum-sensitive tumors are actually platinum-resistant clinically; in these cases, the correct choice of treatment, as indicated by the miniPDX system, would be particularly important. Our results showed that two of the 12 patients (patients 5 and 7) had platinum-resistant "platinum-sensitive recurrent ovarian cancer". After using the regimens recommended by the mini-PDX system, these patients reached a partial response and stable disease, respectively.

However, our new system has certain limitations that also need to be considered. The sensitivity test for the mini-PDX models was established by simulating the use of several regimens on nude mice at the same time. Consequently, the effects of chemotherapeutic regimens that work by regulating the cell cycle may not be reproduced in an acceptable manner. In addition, when the same drug was used in different cycles (such as weekly albumin-bound paclitaxel vs albumin-bound paclitaxel used every 3 weeks), the mini-PDX models could not simulate the actual drug administration and effects since the drugs in mini-PDX models were all used within 7 days. In addition, the use of mini-PDX models is still in its initial phases; further evidence relating to the consistency of clinical outcomes must be acquired. We observed that several nude mice experienced a loss of weight that exceeded $15 \%$ during the testing period; this might explain the grade-III bone marrow depression experienced by patient 5. However, the relevance of the potential relationship between weight loss in the mini-PDX models and the side effects experienced by the matched patients has not yet been proven. 
Table 5 Treatment After Enrollment, Clinical Response, and Side Effects

\begin{tabular}{|c|c|c|c|}
\hline $\begin{array}{l}\text { Patient } \\
\text { Number }\end{array}$ & Chemotherapy Plan After Drug Selection & $\begin{array}{l}\text { Clinical } \\
\text { Outcome }\end{array}$ & Side Effects \\
\hline 1 & $\begin{array}{l}\text { Liposomal doxorubicin*2 (Day I: Liposomal doxorubicin } 60 \mathrm{mg} \text { ), Docetaxel+Carboplatin*4 } \\
\text { (Day I: Docetaxel } 120 \mathrm{mg} \text {, Day 2: Carboplatin } 600 \mathrm{mg} \text { ), Nab-paclitaxel*4 (Day I: Nab- } \\
\text { paclitaxel } 260 \mathrm{mg} \text { ) }\end{array}$ & SD & $\begin{array}{l}\text { Nausea (grade I) } \\
\text { Alopecia (grade I) }\end{array}$ \\
\hline 2 & $\begin{array}{l}\text { Liposomal doxorubicin+Cisplatin*4 } \\
\text { (Day I: Liposomal doxorubicin } 60 \mathrm{mg}+\text { Cisplatin } 60 \mathrm{mg} \text { ) }\end{array}$ & PR & Vomiting (grade I) \\
\hline 3 & $\begin{array}{c}\text { Nab-paclitaxel+ Bevacizumab*4 } \\
\text { (Day I: Nab-paclitaxel } 260 \text { mg+ Bevacizumab } 400 \mathrm{mg} \text { ) }\end{array}$ & PR & $\begin{array}{l}\text { Vomiting (grade I) } \\
\text { Paresthesia (grade } \\
\text { I) }\end{array}$ \\
\hline 4 & $\begin{array}{l}\text { Gemcitabine+Oxaliplatin*2, Pembrolizumab } 200 \text { mg once } \\
\text { (Day I: Gemcitabine } 1200 \text { mg+Oxaliplatin } 100 \text { mg; Day 8: Gemcitabine } 1200 \text { mg) }\end{array}$ & PD & $\begin{array}{l}\text { Nausea (grade II) } \\
\text { Ventosity (grade II) }\end{array}$ \\
\hline 5 & $\begin{array}{c}\text { Liposomal doxorubicin+Topotecan*6 (Day I: Liposomal doxorubicin: } 60 \text { mg Day I-Day 3: } \\
\text { Topotecan } 6 \mathrm{mg} \text { ) }\end{array}$ & PR & $\begin{array}{l}\text { Leukopenia (grade } \\
\text { III) }\end{array}$ \\
\hline 6 & $\begin{array}{c}\text { Apatinib+Etoposide*4 months } \\
\text { (Apatinib: } 750 \mathrm{mg} \text { qd PO, Etoposide: } 75 \mathrm{mg} \text { qd PO*3 weeks, following I week's rest) }\end{array}$ & CR & $\begin{array}{l}\text { Vomiting (grade II) } \\
\text { Alopecia (grade I) }\end{array}$ \\
\hline 7 & $\begin{array}{l}\text { Liposomal dox*4 } \\
\text { (Day I: Liposomal dox } 60 \mathrm{mg} \text { ) }\end{array}$ & SD & $\begin{array}{l}\text { Leukopenia (grade } \\
\qquad \text { II) } \\
\text { Anemia (grade II) }\end{array}$ \\
\hline 8 & $\begin{array}{c}\text { Topotecan*2 } \\
\text { (Day I and Day 8: Topotecan } 5 \mathrm{mg} \text { ) }\end{array}$ & PD & $\begin{array}{l}\text { Leukopenia (grade } \\
\qquad \text { II) } \\
\text { Nausea (grade I) }\end{array}$ \\
\hline 9 & $\begin{array}{l}\text { Topotecan+Bevacizumab*4 } \\
\text { (Day I: Bevacizumab } 400 \mathrm{mg} \text {, Day I, 8, and I5: Topotecan } 4 \mathrm{mg} \text { ) }\end{array}$ & PR & $\begin{array}{l}\text { Vomiting (grade II) } \\
\text { Alopecia (grade I) }\end{array}$ \\
\hline 10 & $\begin{array}{c}\text { Bevacizumab+Olaparib } \\
\text { (Day I: Bevacizumab } 400 \text { mg, Day I-2I: Olaparib } 300 \text { mg bid PO.) }\end{array}$ & PD & $\begin{array}{c}\text { Vomiting (grade I) } \\
\text { Alopecia (grade II) } \\
\text { Thrombocytopenia } \\
\text { (grade II) }\end{array}$ \\
\hline 11 & $\begin{array}{l}\text { Apatinib+etoposide*4 months and continuing until end of follow-up } \\
\text { (Apatinib: } 750 \mathrm{mg} \text { qd PO, Etoposide: } 75 \mathrm{mg} \text { qd PO*3 weeks, following I week of rest) }\end{array}$ & SD & Vomiting (grade I) \\
\hline 12 & $\begin{array}{l}\text { Liposomal dox*4 } \\
\text { (Day I: Liposomal dox } 60 \mathrm{mg} \text { ) }\end{array}$ & PR & Vomiting (grade I) \\
\hline
\end{tabular}

Notes: All drugs were used intravenously if not mentioned otherwise. "* $n$ " refers to the number of cycles of this regimen. The chemotherapy cycle was usually $2 I$ days if not mentioned otherwise. Side effects were graded according to the CTCAEv5.0 standards.

Owing to the time and other restrictions, there are still many improvements that could be made to our model system. Creating a personalized drug-screening system is labor intensive and costly; these issues are further influenced by time limitations and thus led to a small sample size. However, the positive results we obtained from this single-arm trial made it reasonable to anticipate a subsequent clinical trial with an expanded sample size and a longer follow-up time. This type of investigation would also allow us to determine PFS (Progress-free Survival) and OS (Overall Survival) of the patients included and thus help to provide further data relating to how our personalized drug-screening system can influence clinical outcomes. 


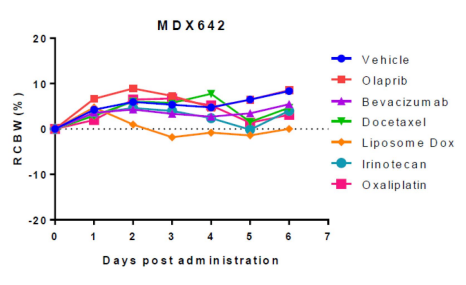

Patient No. 1

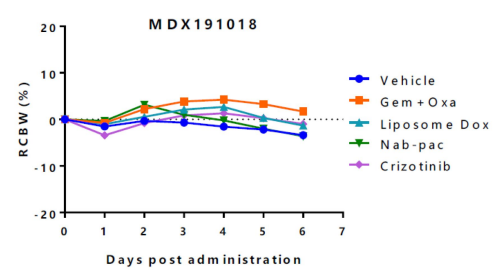

Patient No. 3

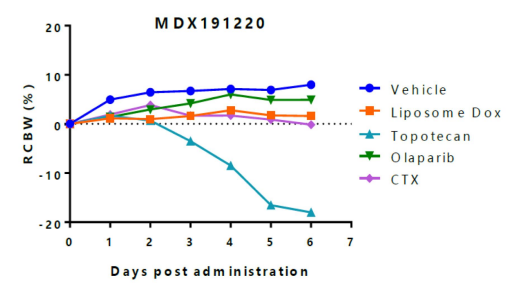

Patient No. 5

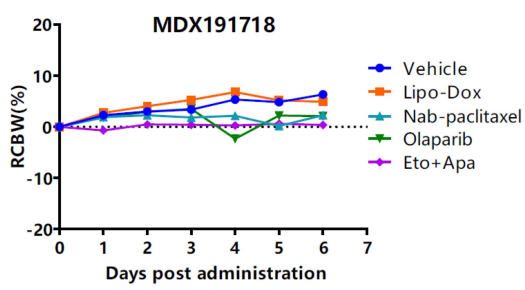

Patient No. 7

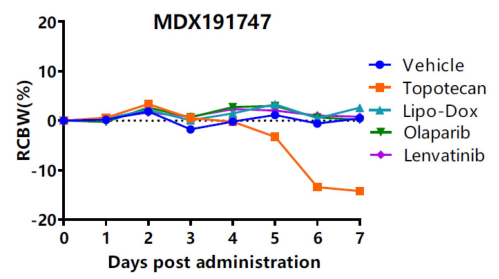

Patient No. 9

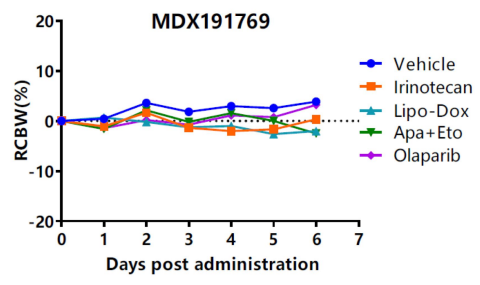

Patient N0.11

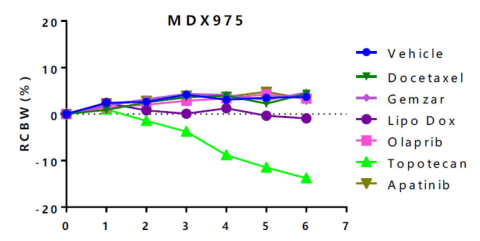

Days post ad $m$ in istration

Patient No. 2

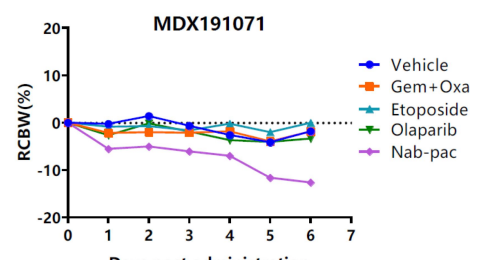

Days post administration

Patient No. 4

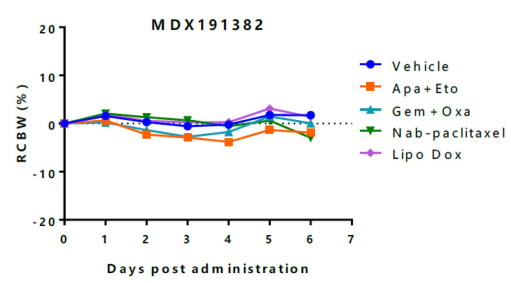

Patient No. 6

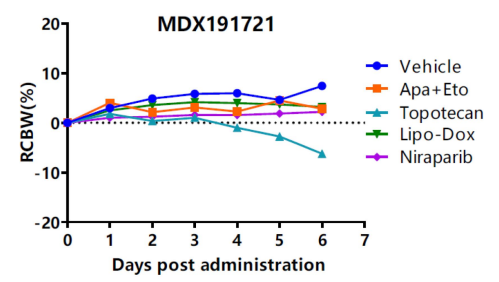

Patient No. 8

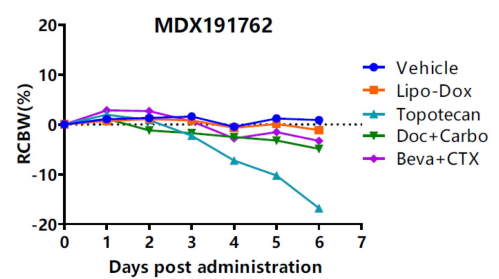

Patient No. 10

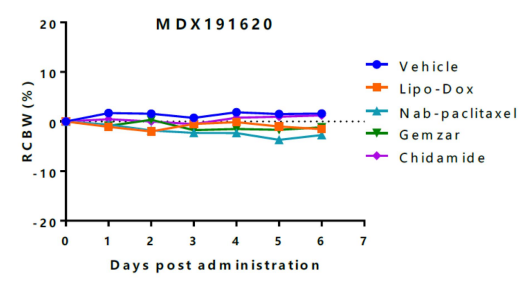

Patient N0.12

Figure 5 The loss of weight in mice during the 7 days of drug treatment. RCBW\% = (BWi-BW0)/BW0*100\%; BWi represents the body weight of the mice on dayl while BW0 represents the body weight of mice when the mini-PDX model was first established. Abbreviations for chemotherapy: Liposomal doxorubicin (Lipo Dox); Nab-pac (Nab-paclitaxel); Gem+Oxa (Gemcitabine+Oxaliplatin); CTX (Cyclophosphamide); Apa+Eto (Apatinib+Etoposide); Doc+Carbo (Docetaxel+Carboplatin); Bev+CTX (Bevacizumab+Cyclophosphamide); Gemzar (Gemcitabine). 


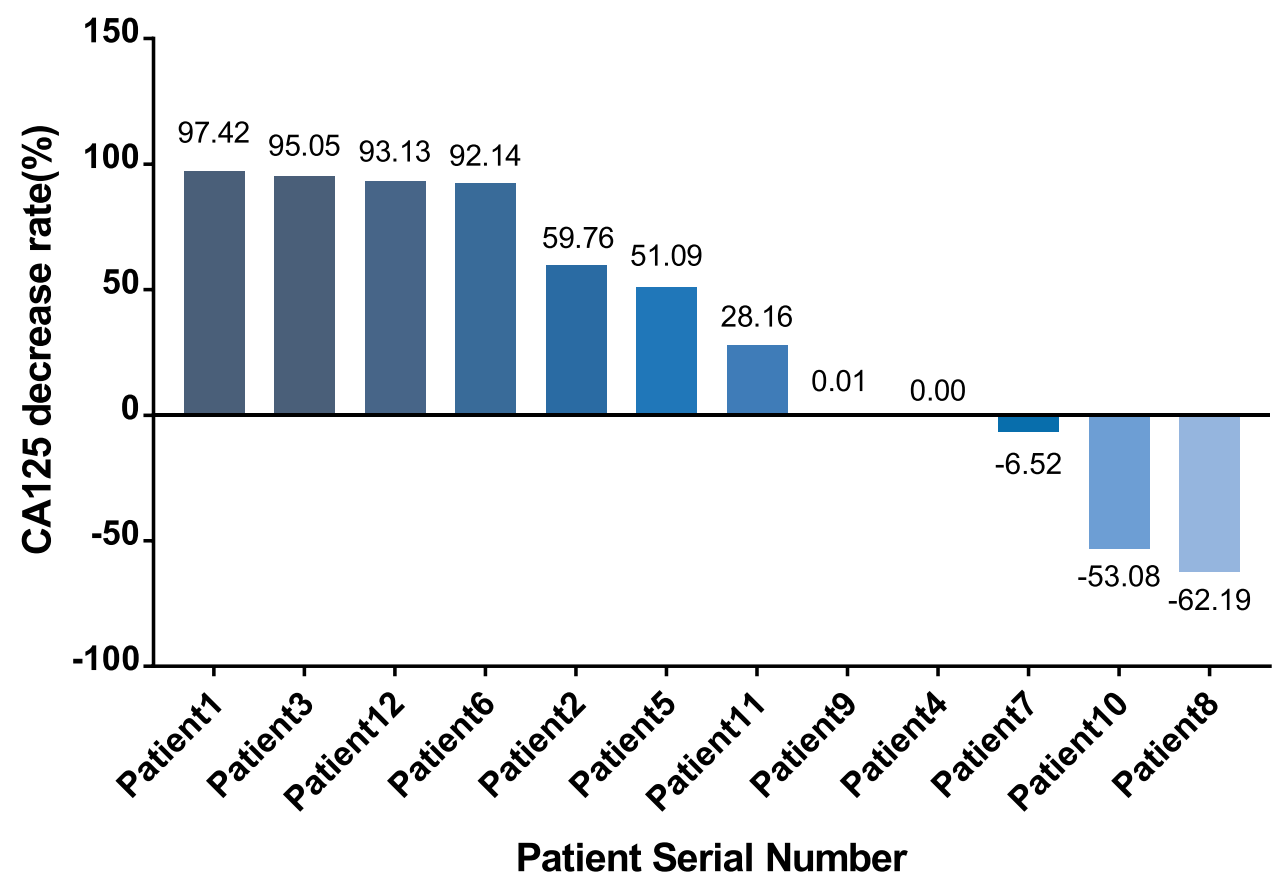

Figure 6 The rate of CAI 25 reduction in the 12 patients. The Y axis shows the rate of reduction in CAI25, as calculated by the following formula: (CAI25 level after all cycles with the observed CAI 25 level prior to the first cycle of the observed regimen)/CAI 25 level prior to the first cycle of the observed regimen*I00\%). This rate was a negative number when the level of CAI 25 increased after treatment. The $X$ axis represents the 12 patients ranked from the highest to the lowest (negative) rate of CAI 25 reduction.

\section{Data Sharing Statement}

The authors (Yunke Huang, yunke_9949@qq.com; Yu Kang, yukang@fudan.edu.cn)would share the clinical data with all the researchers, the clinical trial data (including age, medical history, pathological examinations, imaging examination results, mutation detection results, mini-PDX drug sensitivity results, follow-up results, etc) would be uploaded to ResMan (www.medresman.org) right after publication of the article. The clinical protocol would also be attached for reference.

\section{Ethics Approval}

The present study was approved by the Ethical Committee of the Obstetrics \& Gynecology Hospital of Fudan University (approval number:2016-51-X1).

\section{Consent to Participate}

Written informed consent was provided by all participants before enrollment.

\section{Consent to Publication}

All the authors agree with the publication of this article.

\section{Acknowledgments}

This research was supported by National Key R\&D Program of China:2016YFC1303102 Science and
Technology Commission of Shanghai Municipality: 18140902502; 18411963300 Shanghai Health and Medical Development Foundation: 122018BR26; ZYKC201701020 Shanghai Shenkang Hospital Clinical Development Plan: SHDC12018X13 Lianyu Lin's work on ctDNA tests at the central laboratory of Topgen BioPharm Co, Ltd., sequencing with OncoDrug-SeqTM (Topgen-Biopharm, shanghai, China) was appreciated by all the authors.

\section{Funding}

This work was supported by National Key R\&D Program of China: 2016YFC1303102, Science and Technology Commission of Shanghai Municipality: 18140902502; 18411963300, Shanghai Health and Medical Development Foundation: 122018BR26; ZYKC20170 1020, and Shanghai Shenkang Hospital Clinical Development Plan: SHDC12018X13.

\section{Disclosure}

The authors confirm that there are no conflicts of interest.

\section{References}

1. Siegel RL, Miller KD, Jemal A. Cancer statistics, 2017. CA Cancer J Clin. 2017;67(1):7. 
2. Oronsky B, Ray CM, Spira AI, et al. A brief review of the management of platinum-resistant-platinum-refractory ovarian cancer. Med Oncol. 2017;34(6):103. doi:10.1007/s12032-017-0960-z

3. Ferrero A, Ditto A, Giorda G, et al. Secondary cytoreductive surgery for isolated lymph node recurrence of epithelial ovarian cancer: a multicenter study. Eur J Surg Oncol. 2014;40(7):891-898. doi:10.1016/j.ejso.2013.11.026

4. Colombo N. 327 Randomised trial of paclitaxel in combination with platinum chemotherapy versus platinum-based chemotherapy in the treatment of relapsed ovarian cancer (ICON4/OVAR 2.2). Eur $J$ Cancer. 2003;1(5):S101-S101.

5. Naumann DRW, Coleman RL. Management strategies for recurrent platinum-resistant ovarian cancer. Drugs. 2011;71(11):1397-1412. doi:10.2165/11591720-000000000-00000

6. Heintz A, Odicino F, Maisonneuve $\mathrm{P}$, et al. Carcinoma of the Ovary. Int J Gynecol Obstet. 2006;83(3):p. 135-166. doi:10.1016/S00207292(03)90118-4

7. National Comprehensive Cancer Network. NCCN Clinical Practice Guidelines in Oncology. Ovarian Cancer[EB/OL]; 2020. Available from: https://www.nccn.org/professionals/physician_gls/pdf/ovarian. pdf. Accessed March 19, 2021.

8. Gordon AN, Tonda M, Sun S, et al. Long-term survival advantage for women treated with pegylated liposomal doxorubicin compared with topotecan in a Phase 3 randomized study of recurrent and refractory epithelial ovarian cancer. Gynecol Oncol. 2004;95:1-8. doi:10.1016/j. ygyno.2004.07.011

9. Ferrandina G, Ludovisi M, Lorusso D, et al. Phase III trial of gemcitabine compared with pegylated liposomal doxorubicin in progressive or recurrent ovarian cancer. J Clin Oncol. 2008;26:890-896. doi:10.1200/JCO.2007.13.6606

10. Markman M. Pegylated liposomal doxorubicin: appraisal of its current role in the management of epithelial ovarian cancer. Cancer Manag Res. 2011;3:219-225. doi:10.2147/CMAR.S15558

11. Rose PG, Blessing JA, Mayer AR, et al. Prolonged oral etoposide as second-line therapy for platinum-resistant and platinum-sensitive ovarian carcinoma: a Gynecologic Oncology Group study. J Clin Oncol. 1998;16:405-410. doi:10.1200/JCO.1998.16.2.405

12. Rose PG, Blessing JA, Ball HG, et al. A Phase II study of docetaxel in paclitaxel-resistant ovarian and peritoneal carcinoma: a Gynecologic Oncology Group study. Gynecol Oncol. 2003;88:130135. doi:10.1016/S0090-8258(02)00091-4

13. Hanker LC, Loibl S, Burchardi N, et al. The impact of second to sixth line therapy on survival of relapsed ovarian cancer after primary taxane/platinum-based therapy. Ann Oncol. 2012;23(10):2605-2612. doi:10.1093/annonc/mds203

14. Collins FS, Varmus H, New A. Initiative on precision medicine. New Engl J Med. 2015;372(9):793-795. doi:10.1056/NEJMp15 00523

15. Birgitta S, Gerhards R, Strumberg D, et al. Combined detection of Her $2 /$ neu gene amplification and protein overexpression in effusions from patients with breast and ovarian cancer. $J$ Cancer Res Clin Oncol. 2010;136(9):1389-1400. doi:10.1007/s00432-0100790-2

16. Fujimoto M, Matsuzaki I, Nishino M, et al. HER2 is frequently overexpressed in hepatoid adenocarcinoma and gastric carcinoma with enteroblastic differentiation: a comparison of 35 cases to 334 gastric carcinomas of other histological types. J Clin Pathol. 2018; jclinpath-2017-204928.

17. Gordon MS, Matei D, Aghajanian C, et al. Clinical activity of pertuzumab (rhuMab 2C4) in advanced, refractory or recurrent ovarian cancer (OC), and the role of HER2 activation status. $J$ Clin Oncol. 2005;2(1):35-37.

18. Weroha SJ, Oberg AL, Ziegler KLA, et al. Phase II trial of lapatinib and topotecan (LapTop) in patients with platinum-refractory/resistant ovarian and primary peritoneal carcinoma. Gynecol Oncol. 2011;122 (1):116-120. doi:10.1016/j.ygyno.2011.03.030
19. Aghajanian C, Blank SV, Goff BA, et al. OCEANS: a randomized, double- blind, placebo controlled phase III trial of chemotherapy with or without bevacizumab in patients with platinum-sensitive recurrent epithelial ovarian, primary peritoneal, or fallopian tube cancer. J Clin Oncol. 2012;30(17):2039-2045. doi:10.1200/JCO.2012.42.0505

20. Pujade-Lauraine E, Hilpert F, Weber B. AURELIA: a randomized phase III trial evaluating bevacizumab (BEV) plus chemotherapy (CT) for platinum (PT)-resistant recurrent ovarian cancer (OC). $J$ Clin Oncol. 2012;30(15_suppl):LBA5002-LBA5002. doi:10.1200/ jco.2012.30.15_suppl.1ba5002

21. Ray-Coquard IL, Pautier P, et al. Phase III PAOLA-1/ENGOT-ov25 trial: olaparib plus bevacizumab (bev) as maintenance therapy in patients (pts) with newly diagnosed, advanced ovarian cancer (OC) treated with platinum-based chemotherapy (PCh) plus bev. presented at ESMO Annual Conference 2019, 27 September - 1 October. Barcelona, Spain.

22. Scott CL, Becker MA, Haluska P, et al. Patient -derived xenograft models to improve targeted therapy in epithelial ovarian cancer treatment. Front Oncol. 2013;3:295. doi:10.3389/fonc.2013.00295

23. Topp MD, Hartley L, Cook M, et al. Molecular correlates of platinum response in human high-grade serous ovarian cancer patient-derived xenografts. Mol Oncol. 2014;8(3):656-668. doi:10.1016/j. molonc.2014.01.008

24. Letai A. Functional precision cancer medicine - moving beyond pure genomics. Nat Med. 2017;23(9):1028-1035. doi:10.1038/nm.4389

25. Pauli C, Hopkins BD, Prandi D, et al. Personalized in vitro and in vivo cancer models to guide precision medicine. Cancer Discov. 2017;7(5):462. doi:10.1158/2159-8290.CD-16-1154

26. Friedman AA, Letai A, Fisher DE, et al. Precision medicine for cancer with next-generation functional diagnostics. Nat Rev Cancer. 2015;15(12):747-756. doi:10.1038/nrc4015

27. Hidalgo M, Amant F, Biankin AV, et al. Patient-derived xenograft models: an emerging platform for translational cancer research. Cancer Discov. 2014;4(9):998-1013. doi:10.1158/2159-8290.CD-14-0001

28. Zhang F, Wang W, Long Y, et al. The establishment of mini-PDX models for predicting the clinical treatment plan. Chin J Cancer. 2019;4.

29. Ming Z, Rui-Meng Y, Hui W, et al. Guided chemotherapy based on patient-derived mini-xenograft models improves survival of gallbladder carcinoma patients. Cancer Commun. 2018;38(1):48. doi:10.11 86/s40880-018-0318-8

30. Eisenhauer EA, Therasse P, Bogaerts J, et al. New response evaluation criteria in solid tumours: revised RECIST guideline (version 1.1). Eur J Cancer. 2009;45:228-247. doi:10.1016/j.ejca.2008.10.026

31. Burger RA, Sill MW, Monk BJ, et al. Phase II trial of bevacizumab in persistent or recurrent epithelial ovarian cancer or primary peritoneal cancer: a Gynecologic Oncology Group Study. J Clin Oncol. 2007;25:5165.

32. Chun-Yan L, Yin W, Ying X, et al. Apatinib combined with oral etoposide in patients with platinum-resistant or platinum-refractory ovarian cancer (AEROC): a Phase 2, single-arm, prospective study. Lancet Oncol. 2018;S147020451830349-8.

33. Domchek SM, Aghajanian C, Shapira-Frommer R, et al. Efficacy and safety of olaparib monotherapy in germline BRCA1/2 mutation carriers with advanced ovarian cancer and three or more lines of prior therapy. Gynecol Oncol. 2015;S0090825815302237.

34. Dal MGZ, Westin SN, Coleman RL. Rucaparib in ovarian cancer: extending the use of PARP inhibitors in the recurrent disease. Future Oncol. 2018;fon-2018-0215.

35. Moore KN, Secord AA, Geller MA, et al. Niraparib monotherapy for late-line treatment of ovarian cancer (QUADRA): a multicentre, open-label, single-arm, phase 2 trial. Lancet Oncol. 2019;20 (5):636-648. doi:10.1016/S1470-2045(19)30029-4

36. Sicklick JK, Kato S, Okamura R, et al. Molecular profiling of cancer patients enables personalized combination therapy: the I-PREDICT study. Nat Med. 2019;25(5):744-750. doi:10.1038/ s41591-019-0407-5 
37. Heo EJ, Cho YJ, Cho WC, et al. Patient-derived xenograft models of epithelial ovarian cancer for preclinical studies. Technol Cancer Res T. 2017;49(4):915-926. doi:10.4143/crt.20 16.322
38. Ricci F, Guffanti F, Damia G, et al. Combination of paclitaxel, bevacizumab and MEK162 in second line treatment in platinumrelapsing patient derived ovarian cancer xenografts. Mol Cancer. 2017;16(1):97. doi:10.1186/s12943-017-0662-3

\section{Publish your work in this journal}

Cancer Management and Research is an international, peer-reviewed open access journal focusing on cancer research and the optimal use of preventative and integrated treatment interventions to achieve improved outcomes, enhanced survival and quality of life for the cancer patient.
The manuscript management system is completely online and includes a very quick and fair peer-review system, which is all easy to use. Visit http://www.dovepress.com/testimonials.php to read real quotes from published authors. 